\title{
On the nightglow polarisation for space weather exploration
}

\author{
Léo Bosse ${ }^{1, *}$, Jean Lilensten ${ }^{1,5}$, Nicolas Gillet ${ }^{2}$, Sylvain Rochat ${ }^{1}$, Alain Delboulbé ${ }^{1}$, Stephane Curaba ${ }^{1}$, \\ Alain Roux ${ }^{1}$, Yves Magnard ${ }^{1}$, Magnar G. Johnsen ${ }^{4}$, Unni-Pia Løvhaug ${ }^{6}$, Pierre-Olivier Amblard ${ }^{3}$, \\ Nicolas Le Bihan ${ }^{3}$, Maxime Nabon ${ }^{1}$, Hanane Marif ${ }^{7}$, Frédérique Auriol ${ }^{8}$, and Camille Noûs ${ }^{9}$ \\ ${ }^{1}$ Institut de Planétologie et d'Astrophysique de Grenoble (IPAG) CNRS - UGA, France \\ ${ }^{2}$ Univ. Grenoble Alpes, Univ. Savoie Mont Blanc, CNRS, IRD, IFSTTAR, ISTerre, 38000 Grenoble, France \\ ${ }^{3}$ GIPSA-lab, Dept. Images and Signals, UMR CNRS, France \\ ${ }^{4}$ Tromsø Geophysical Observatory, UiT - the Arctic University of Norway, Troms $\varnothing$, Norway \\ ${ }^{5}$ Honorary Astronomer at Royal Observatory of Belgium, Brussels \\ ${ }^{6}$ Department of Physics and Technology, UiT - the Arctic University of Norway, Troms $\varnothing$, Norway \\ ${ }^{7}$ University of Science and Technology Houari Boumediene - USTHB, Faculty of Physics \\ ${ }^{8}$ Univ. Lille, CNRS, UMR 8518 - LOA - Laboratoire d'Optique Atmosphérique, 59000 Lille, France \\ ${ }^{9}$ Laboratoire Cogitamus, France
}

Received 25 February 2020 / Accepted 30 June 2020

\begin{abstract}
We present here observations of the polarisation of four auroral lines in the auroral oval and in the polar cusp using a new ground polarimeter called Petit Cru. Our results confirm the already known polarisation of the red line, and show for the first time that the three other lines observed here (namely $557.7 \mathrm{~nm}, 391.4 \mathrm{~nm}$ and $427.8 \mathrm{~nm}$ ) are polarised as well up to a few percent. We show that in several circumstances, this polarisation is linked to the local magnetic activity and to the state of the ionosphere through the electron density measured with EISCAT. However, we also show that the contribution of light pollution from nearby cities via scattering can not be ignored and can play an important role in polarisation measurements. This series of observations questions the geophysical origin of the polarisation. It also leaves open its relation to the magnetic field orientation and to the state of both the upper atmosphere and the troposphere.
\end{abstract}

Keywords: polarisation / aurora

\section{Introduction}

Auroral lights are mainly triggered by collisions between electrons and atoms or molecules in the upper atmosphere. The de-excitation of ionospheric (i.e. ions and charged particles) and thermospheric (i.e. neutral particles) generates emissions in many wavelengths. The most prominent being the red, green, blue and purples wavelengths (see Table 1). Duncan (1959) predicted and observed the polarisation of the auroral red line emissions near Sydney, Australia. His results were quickly refuted by Chamberlain (1959), invoking depolarisation through collisions. Attempts to measure the auroral emission polarisation were attempted from the Auroral Observatory in Troms $\varnothing$, Northern Norway, by Harang (1933) and Harang (1960). In both cases results proved negative, most likely owing to low sensitivity in the instrumentation. Further attempts were left aside until the beginning of the 21st century. Lilensten et al. (2006) reconsidered this problem and found out that even though a polarisation of $30 \%$ as measured by Duncan (1959) seems too high, the refutation by Chamberlain might not fully apply, and that a few percent of polarisation may remain.

A Spectro-Photo-Polarimeter (SPP) was built with the aim of measuring this polarisation from Svalbard Lilensten et al. (2008). The first results were in agreement with the theoretical work of Bommier et al. (2011), allowing a few percent of polarisation in the red line. Indeed, auroral electrons, coming from a preferential direction along the geomagnetic field lines can trigger a dipolar transition of atomic oxygen $\mathrm{O}$, emitting red light polarised parallel to the incoming direction. Incidentally, this work also predicts no polarisation in the green line $(557.7 \mathrm{~nm}$, see Table 1), this latter being associated with a quadrupolar transition of $\mathrm{O}$, thus forbidding polarisation.

Through a series of campaigns, several characteristics of the auroral red line polarisation have been revealed.

*Corresponding author: leo.bosse@univ-grenoble-alpes.fr 
Table 1. The four main auroral lines studied in this paper and their properties. The width in column 5 corresponds to the spectral size of the instrument interference filters and not to the upper atmospheric emission.

\begin{tabular}{lccccc}
\hline Altitude $(\mathrm{km})$ & Colour & Element & Transition & Wavelength $(\mathrm{nm})$ & Energy threshold $(\mathrm{eV})$ \\
\hline 220 & Red & $\mathrm{O}$ & $1 \mathrm{D} \rightarrow 3 \mathrm{P}$ & $630 \pm 1$ & 1.96 \\
110 & Green & $\mathrm{O}$ & $1 \mathrm{~S} \rightarrow$ 1D & $557.7 \pm 5$ & 4.00 \\
80 & Blue & $\mathrm{N}_{2}^{+}$ & 1 st negative band & $427.8 \pm 5$ & 18.75 \\
80 & Purple & $\mathrm{N}_{2}^{+}$ & 1 st negative band & $391.4 \pm 5$ & 18.75 \\
\hline
\end{tabular}

In Lilensten et al. (2016), a Degree of Linear Polarisation (DoLP) between $1 \%$ and $12 \%$ has been observed in the auroral red line, with an average of about $5 \%$. It is anti-correlated with the line intensity, which was interpreted as an increase in collisions during the aurora. An absolute measure of the Angle of Linear Polarisation (AoLP) was not available with SPP. Nevertheless, variations in the orientation of the observed polarisation were visible, and linked to changes in the DoLP. Following a first theoretical study, the contribution of light pollution from cities and aerosols in the lower atmosphere was considered as negligible (Barthélémy et al., 2011), and polarisation was attributed to upper atmospheric phenomena.

The limitations of the SPP instrument were strong. First it was difficult to access a calibrated angle of polarisation. Second, the instrument was not steerable, making it difficult to sample various azimuths and elevations. Therefore, a new polarimeter called Petit Cru was built. It gains in precision, with more reliable measurements, a faster sampling rate and an increased sensitivity in the four wavelengths of interest. It is able to detect very faint airglow and has been calibrated for DoLP values as low as $1 \%$. It also provides an absolute measure of the AoLP, allowing to compare the polarisation orientation with that of geomagnetic field lines. The data processing of Petit Cru is based on a lock-in amplifier method (instead of a Fourier analysis for SPP), rendering this new instrument both faster (with real time acquisition and visualisation) and more reliable. Observations that were unreachable before with instruments like SPP are now possible with Petit Cru. This paper reports on some unexpected new findings in the polar and auroral zones. In particular we show links between polarised lights properties and independent proxies of the geophysical activity. We also revisit the importance of processes associated with scattering in the lower atmosphere.

We first introduce in Section 2.1 the Petit Cru instrument along with the methodology used to recover the properties of polarised light (Sect. 2.2). We also detail the principles for the calculation of the apparent angles of the magnetic field on the instrument (Sect. 2.3) and of the angle polarisation for a Rayleigh scattered light from a point source (Sect. 2.4). We then show our main results (Sect. 3), followed by an extended discussion of their implications (Sect. 4) and by concluding remarks (Sect. 5).

\section{Method and instruments}

\subsection{Petit Cru principles}

Petit Cru is more than a simple follow on version of the SPP instrument. All the acquisition chain has been reconsidered even if the basic principle remains the same. In the case of Petit Cru, the incoming light first passes through an angle-calibrated polarising filter with an adaptable rotation velocity tunable between 0.5 and $2 \mathrm{~Hz}$. It allows averaging over $2 \mathrm{~s}$ in case of a clear night with no aurora in order to still observe the nightglow. It also allows to rotate fast enough so that the auroral conditions may be considered as stationary through one rotation of the polarising filter. It also aims at allowing very fast observations to monitor the dynamics of an aurora if necessary. But turning too fast increases the noise in the measurements. In this article, we use a rotation frequency of $2 \mathrm{~Hz}$. It is a good compromise, catching fast dynamics and allowing signal to noise enhancement by smoothing. The data processing allows to pile up several rotations before determining the polarisation parameters. For the sake of clarity, we chose to compute these parameters on 30 rotations $(15 \mathrm{~s})$ for all the data presented in this paper. We checked that this choice does not change any of the features presented in the sequel.

An interference filter selects a wavelength range (any range may be used). Filter properties vary with the temperature, and we avoid measuring a mixture of emissions by using narrow filters. The best way to compensate for such temperature variations is to tilt the filter. A careful calibration (with a calibration lamp) allows to correct this tilt with sufficient precision. We observe the main four auroral lines with interference filters at $630.0 \pm 1 \mathrm{~nm}$ (red), $577.7 \pm 5 \mathrm{~nm}$ (green), $427.8 \pm 5 \mathrm{~nm}$ (blue) and $391.4 \pm 5 \mathrm{~nm}$ (purple) - see details in Table 1. The thinner \pm 1 bandwidth at $630 \mathrm{~nm}$ is chosen because the red line is a triplet with emissions at 630.0, 636.4 and $639.2 \mathrm{~nm}$. Such a narrow bandwidth allows to rule out any overlapping with the $636.4 \mathrm{~nm}$ emission.

Finally, a Photo-Multiplier (PM) samples the intensity behind these two filters at $1000 \mathrm{~Hz}$ with 24 bits. The quantum efficiency of the detector is $40 \%$. The entire wavelength range of all filters have been compared to the photomultiplier sensitivity range to ensure no out of band radiations are detected. The objective lens has a $50 \mathrm{~mm}$ aperture and a $150 \mathrm{~mm}$ focal length. A diaphragm controls the aperture of the beam line, fixed at $2^{\circ}$ in this work. The aperture is defined by a compromise between the signal received and the observed volume. $2^{\circ}$ ensure the observed volume does not span over more than $10 \mathrm{~km}$ and can be supposed homogeneous. The optical design of Petit Cru, together with an on-site picture of the instrument, are shown in Figure 1. The whole optical axis is isolated from the polarising filter to the photomultiplier to ensure no internal reflections of light from outside the field of view. This instrument is low-consumption ( $>20 \mathrm{~W}$ ), weights only $4 \mathrm{~kg}$ and fits on a camera tripod.

As opposed to the SPP case, we do not count the photons but we directly measure the output current. If the light is not 


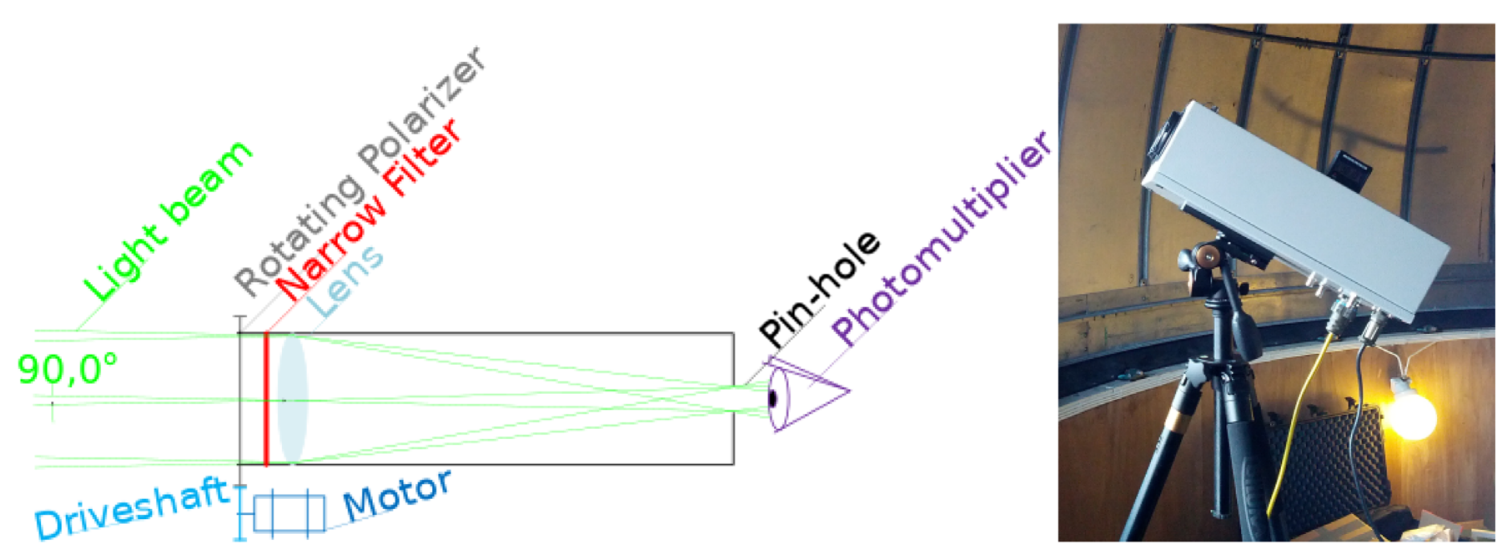

Fig. 1. Optical design (left) and on-site picture (right) of the Petit Cru polarimeter.

polarised, then the recorded intensity does not vary over one rotation of the polarising filter. Alternatively, if it is polarised, we see two maxima, and two minima on the light curve. From the phase and amplitude of these variations, one can retrieve the intensity, the DoLP and the AoLP of the incoming light. The lock-in amplifier method (see Appendix A) allows to analyse the signal in real-time.

Petit Cru was calibrated at the Laboratoire d'Optique Atmosphérique (LOA) in Lille (France) with a dedicated calibration facility (Auriol et al., 2008). A "polarising box" is placed in front of an integrating sphere delivering natural, unpolarised light. It controls the DoLP between $0.3 \%$ and $60 \%$ by tilting two parallel glass slides of known refractive index. The absolute accuracy of the calibration system is estimated to be $0.5 \%$ in DoLP. The theoretical DoLP produced by the system is then compared to the measured DoLP of Petit Cru. The behaviour of the DoLP measurements is similar in the four wavelengths. When smoothed over $15 \mathrm{~s}$, the measured DoLP tends to minimize the calibration DoLP by a factor 0.01 , and can be trusted for DoLP as low as $0.5 \%$. Instrumental polarisation was also tested by rotating the instrument around the optical axis, which does not change the results significantly.

We also checked that Petit Cru does not produce any instrumental polarisation. For this, we used two different methods. In the first one, we take off the polarising filter and check that the DoLP is null. In the second method, we use two parallel channels observing the same polarised source, one with a rotating polarising filter and the other without. We could then insure that no instrumental polarisation was created.

Finally, an inter-comparison between SPP and Petit Cru has been performed in laboratory conditions and during a field campaign on the red line (the only one observable with SPP). Both instruments give the same DoLP value (within 1\%) when Petit Cru is degraded to the same sampling rate as SPP - a relatively stronger dynamics is found at high frequencies when Petit Cru is used at its full speed, with no bias on the average measurements. The DoLP and AoLP are stable with Petit Cru once calibrated. SPP provides the same AoLP when a calibration is performed, but this absolute angle is lost as soon as a shutdown is made. As a consequence, and contrarily to Petit $C r u$, only relative changes in the AoLP can be used with SPP.

When the DoLP is low (below 1\%), the AoLP becomes noisy. The spread of the AoLP depends on the sampling rate and how many polarising filter rotations are used to smooth the data. At $2 \mathrm{~Hz}$, a DoLP of $1 \%$ means a spread over more than $50^{\circ}$ in the AoLP, rendering it unreadable. But smoothed over $15 \mathrm{~s}$, a DoLP as low as $0.5 \%$ induces a maximum spread of $10^{\circ}$. See Appendix A for details on the uncertainty computations.

\subsection{Data processing}

SPP used a Fourier transform based analysis to retrieve the polarisation properties from the intensity time series. Petit $\mathrm{Cru}$ now uses a digital lock-in amplifier method, detailed in Appendix A. It has the advantage of being faster, allowing real-time computations. It is also more accurate. This way, only three values are stored in the data base for each rotation of the polarising filter, instead of a thousand.

Here is a brief overview of the process. We consider an incoming polarised light beam of intensity $I_{0}$ and DoLP between 0 and 1 (scaled from $0 \%$ to $100 \%$ in all the graphs of this work) forming an angle AoLP with the vertical $\left(0^{\circ}\right.$ being upward, $180^{\circ}$ downward). $\theta_{t}$ is the angle of the polarising filter with the vertical at time $t$. We suppose $I_{0}$, DoLP and AoLP to be stationary over one rotation period of the polarising filter $\left(T_{0}\right.$ is half a second in the current work). The line intensity can be decomposed in two parts: a polarised one which varies as a square cosine of the angle of the rotating polarising filter and an unpolarised part, assumed constant over one rotation. For a total intensity $I_{0}$, the polarised intensity is $I_{0}^{\text {pola }}=\operatorname{DoLP} \times I_{0}$ and the unpolarised intensity $I_{0}^{\text {unpola }}=(1-$ DoLP $) \times I_{0}$.

Passing through the polarising filter, Malus Law predicts that the polarised light intensity becomes $I_{t}^{\text {pola }}=$ $I_{0}^{\text {pola }} \cos ^{2}\left(\theta_{t}-\right.$ AoLP $)$, while the unpolarised intensity is $I_{t}^{\text {unpola }}=I_{0}^{\text {unpola }} / 2$. The intensity at any given time $t$ can therefore be written as:

$$
\begin{aligned}
I_{t} & =I_{t}^{\text {pola }}+I_{t}^{\text {unpola }} \\
& =I_{0}\left(\operatorname{DoLP} \times \cos ^{2}\left(\theta_{t}-\mathrm{AoLP}\right)+\frac{1-\mathrm{DoLP}}{2}\right) .
\end{aligned}
$$

This allows computing

$$
V=\frac{1}{T_{0}} \int_{0}^{T_{0}} I_{t} \mathrm{~d} t
$$




$$
\begin{gathered}
V_{c}=\frac{1}{T_{0}} \int_{0}^{T_{0}} I_{t} \cos 2 \theta_{t} \mathrm{~d} t \\
V_{s}=-\frac{1}{T_{0}} \int_{0}^{T_{0}} I_{t} \sin 2 \theta_{t} \mathrm{~d} t .
\end{gathered}
$$

Injecting equation (1) in equations (2)-(4), one deduces the following properties of the incoming light:

$$
\begin{gathered}
I_{0}=2 V, \\
\operatorname{DoLP}=\frac{2}{V} \sqrt{V_{c}^{2}+V_{s}^{2},} \\
\operatorname{AoLP}=\frac{1}{2} \arctan \left(\frac{V_{s}}{V_{c}}\right) .
\end{gathered}
$$

To smooth the data over several polarising filter rotations, the correct mathematical way is to average $V, V_{c}$ and $V_{\mathrm{s}}$ values over the desired number of rotations and then to calculate the corresponding polarisation properties. Post-averaging the DoLP and AoLP would lead to biased estimators that should not be used (see Appendix A).

\subsection{Apparent angle of the magnetic field lines}

Lilensten et al. (2016) suggested that the polarisation direction of the auroral red line is parallel to the general incoming direction of the electrons, i.e., globally parallel to the magnetic field lines. No measurements of the local magnetic field are available for these altitudes ( $80-300 \mathrm{~km})$, but there exist different global models derived from magnetic data. To compare the measured AoLP with the apparent angle of the geomagnetic field, we use the internal magnetic field as modeled by the IGRF (Thébault et al., 2015) or by CHAOS-6 (Finlay et al., 2016). Both models lead to very similar apparent angles and are used indifferently. We neglect here the contribution from external magnetic sources. These can change locally the orientation of the total field by a few degrees, but are difficult to accurately recover in auroral regions (e.g. Finlay et al., 2017). We project the apparent field line at the emission point onto the instrument plane (see Appendix B for details). We can then compare its orientation with the measured AoLP.

\subsection{Rayleigh scattering model}

Even in the most remote place, it is very hard to get rid of all light pollution. When this light is scattered in the troposphere, it gets polarised by Rayleigh mechanisms (Bohren \& Huffman, 2008), and may project onto our AoLP and DoLP measurements. In order to evaluate such effects, we consider an unpolarised and isotropic point source at a given distance of the instrument, and compute the AoLP observed by the instrument when this light is scattered in the line of sight. For Rayleigh scattering, the direction of polarisation is perpendicular to the plane defined by the incoming and scattered light trajectories. By construction, this plane can also be defined by the line of sight of the instrument and the direction of the point source. Note that the distance to the source does not matter for the computation of the angle and neither does the point of scattering along the line of sight. Of course, these parameters are very important to compute the scattered intensity and DoLP, but this would require a much more complicated model, which is out of the scope of the present study. We refer to Appendix C for the calculations details.

The above framework allows to compare the observed AoLP in simple configurations. This rough estimate of the AoLP is only valid for a localized light pollution source, and does not give access to the DoLP, nor to the intensity of the scattered light. Note finally that such Rayleigh polarisation may also be important when considering an aurora outside the aperture of the beam line, of which the light is scattered in the lower atmosphere and enters the Petit Cru instrument. In such a case one would need to abandon the hypothesis of a localized source.

\section{Results}

In this paper, we focus on two campaigns during winter 2018-2019 at the Skibotn Astronomical Observatory in Norway $\left(69.34812^{\circ} \mathrm{N}, 20.36322^{\circ} \mathrm{E}\right)$ and in Ny Alesund, Svalbard $\left(78.92324^{\circ} \mathrm{N}, 11.92286^{\circ} \mathrm{E}\right)$. The Skibotn observation site is located $5 \mathrm{~km}$ South-West from the village. In Ny Alesund, the instrument is installed on the roof of a building on the South side of the village. Skibotn is located in the auroral zone while Ny Alesund is within the polar cap. Table 2 summarizes the observations considered in this paper. They are taken out of a larger database for being representative of the different behaviors observed, and for summarizing the issues that they raise.

\subsection{The polarisation as a tracer of the geophysical activity}

We first discuss observations corresponding to cases A to D in Table 2, i.e., in clear sky conditions, with visible auroras, and during which EISCAT and magnetometer data are available. EISCAT is an incoherent scatter radar (Baron, 1986) located close to Troms $\varnothing$ (Norway, $69.5866^{\circ} \mathrm{N}, 19.2256^{\circ} \mathrm{E}$ ). It is pointing towards the observed emission point. We use it to evaluate the state of the upper atmosphere in the line of sight at the time of our measurements. In particular, it monitors the auroral activity via the electron density. We use this information to check whether or not our polarisation measurements are linked to ionospheric phenomena. We also use ground-based magnetometers that monitor changes in the magnetic activity. Indeed, auroras are most often associated with sudden changes in the magnetic field (Meek, 1953). We use here the records from the Troms $\varnothing$ Geophysical Observatory (Johnsen, 2013).

We show in Figure 2 observations corresponding to the four emission lines. For each of them, we show three panels, namely time series of the intensity (top), the DoLP (middle) and the AoLP (bottom). In the top panel, we also show when available the electron density measured by EISCAT at the altitude where the line is emitted (cf. Table 1). In the middle panel, we superpose to the DoLP the rate of change of the magnetic field measured in Troms $\varnothing$. We also show in the bottom panels the apparent angle of the magnetic field lines, and the expected angle of polarisation of a scattered light coming from a point source located in the nearest village. For the Skibotn site, 
Table 2. List of all observations shown in this work along with parameters and geophysical conditions. This selection represents a subset of our database, pertinent with our understanding and conclusions. "Alm" stands for "Almucantar" which is a circle of constant elevation in the sky. In this case, we simply perform a full rotation at a constant elevation. f10.7 and Ap values are taken respectively from the Space Weather Canada (https://www.spaceweather.gc.ca/solarflux/sx-en.php) and the International Service of Geomagnetic Indices websites (http://isgi.unistra. fr/data_plot.php). The last column (Atmospheric conditions) is based on our eye observations, on the all-sky camera archives available here: http://tid.uio.no/plasma/allsky_camera, on the local weather bulletins and when available on the analysis of the local lidar in Ny Alesund.

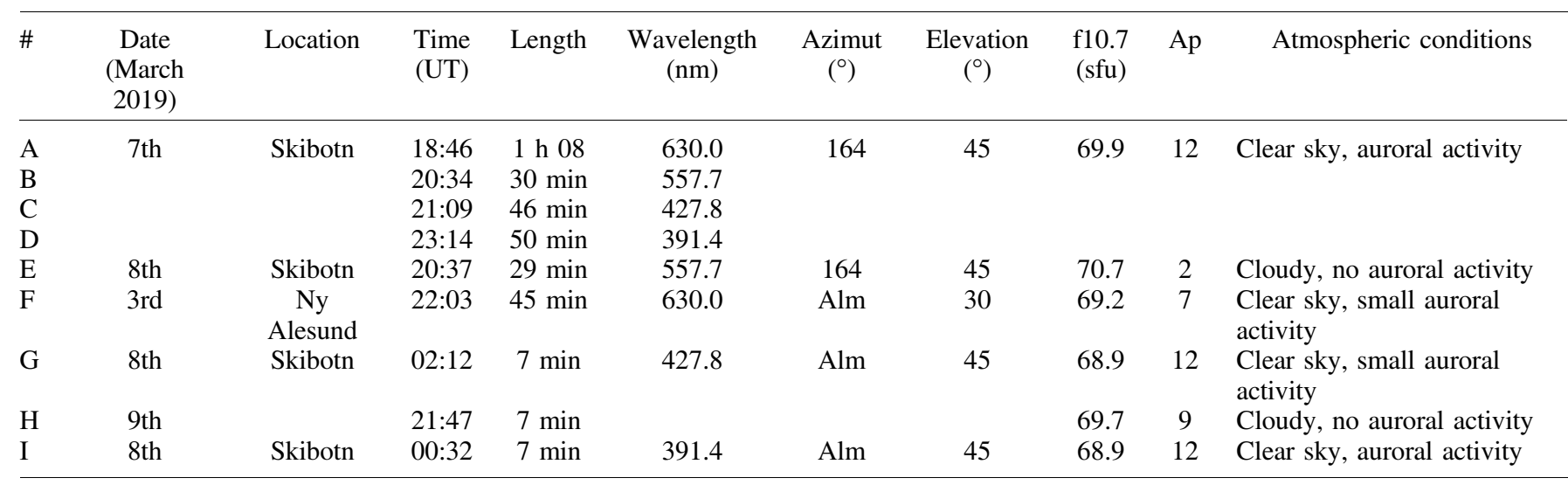

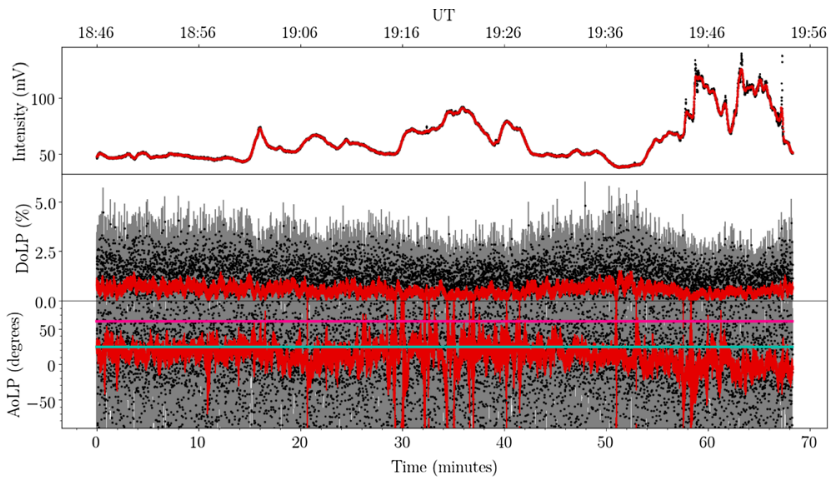

Red line observation $(630.0 \mathrm{~nm})$

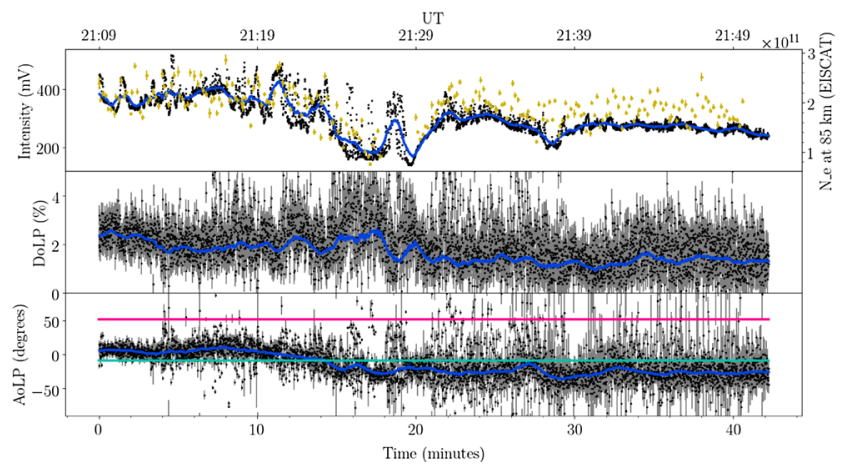

Blue line observation $(427.8 \mathrm{~nm})$

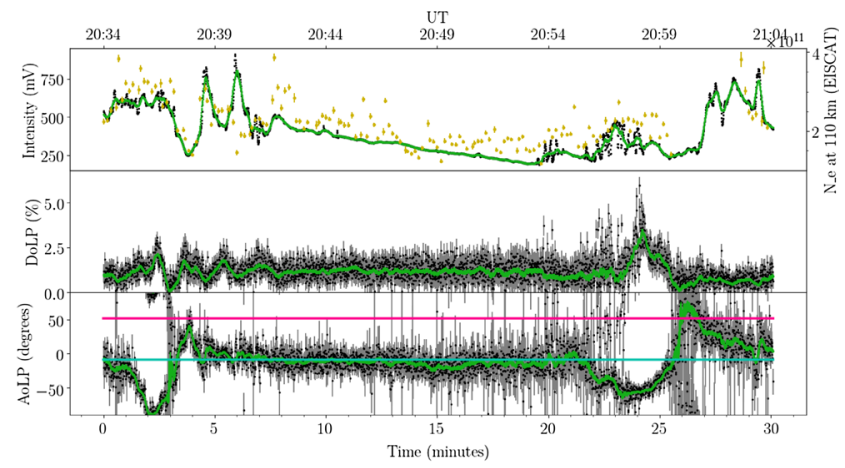

Green line observation $(557.7 \mathrm{~nm})$

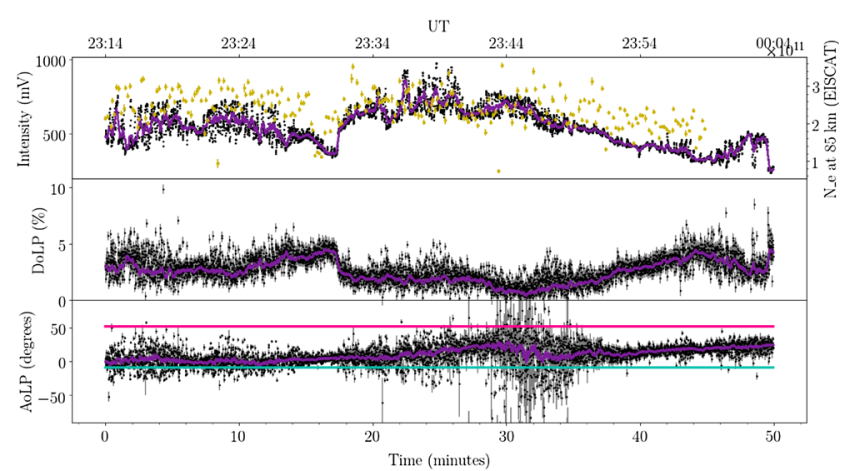

Purple line observation $(391.4 \mathrm{~nm})$

Fig. 2. Polarisation as observed in four wavelengths near Skibotn, Norway on March 7, 2019. Petit Cru is at $164^{\circ}$ in azimuth and $45^{\circ}$ in elevation. Several filters were used to observe the red (top left), green (top right), blue (bottom left) and purple (bottom right) lines. In black are shown the raw data along with the associated error bars in grey. The color lines (red, green, blue, purple) show the corresponding observed wavelengths smoothed over $15 \mathrm{~s}$ and their error bars in the same color. For all 4 wavelengths are given (from top to bottom) the observed intensity (in $\mathrm{mV}$ ), the DoLP (in \%) and the AoLP (in degrees). The intensity can not be compared between two different colors (due to different filters). When available, electron density at the point of emission is shown in yellow on top of the intensity. The rate of change of the horizontal component of the magnetic field in Troms $\varnothing$ is given in yellow on top of the DoLP. We also show, superimposed with the AoLP, the apparent angle of the magnetic field lines (in cyan) and the expected AoLP for scattered light for a artificial source point in Skibotn (in pink). 
we locate the point source at azimuth $-45^{\circ}$ (North-West) and elevation $0^{\circ}$. For Ny Alesund, the point source has azimuth $0^{\circ}$ (North) and elevation $0^{\circ}$. Since the AoLP is cyclic in the interval $\left[-90^{\circ} ; 90^{\circ}\right]$, error bars extending outside the visible range of the graph are reported by hand on the other side of the interval for every figure of the present work.

The red line (Fig. 2, top left) is observed at the beginning of the night, with EISCAT data not yet available. The DoLP, here always around $0.5 \%$, is significantly weaker than in previous measurements by Lilensten et al. (2016) with SPP. We can nevertheless see small increase up to $1 \%$ around 19:36 UT. The magnetometer in Troms $\varnothing$ does not record large variations $(<0.5 \mathrm{nT} / \mathrm{s})$ compared to the rest of the night, but bright auroras were visible all over the sky between $19 \mathrm{~h}$ and $20 \mathrm{~h} \mathrm{UT}$, which can be seen in the doubling of the intensity. The raw AoLP is not defined due to the low DoLP as discussed in Section 2.1 and Appendix A. Once smoothed over $15 \mathrm{~s}$, it is however centered on the apparent angle of the magnetic field lines $\left(<50^{\circ}\right.$ away), and does not correspond to the angle expected from Rayleigh scattering with a source located over the village of Skibotn.

Observations in the green line (Fig. 2, top right) record two short auroral events in the line of sight, which occur during the first and last $10 \mathrm{~min}$, with a low activity in between. These are visible in both the variations of the intensity, the electron density and the magnetometer data. One can note the correlation between the observed intensity and the electron density as measured by EISCAT, which confirms a thermospheric contribution in the data. However, it does not rule out the possibility of a constant scattered light contribution in the foreground. The DoLP is significant, with values as high as $4 \%$, i.e., far above the calibration threshold. This raises an important question since this line is theoretically not polarisable (Bommier et al., 2011). Fluctuations in the DoLP are seen during both events, while a stable DoLP around $1 \%$ is found during the quiet period in between. The AoLP evolves in a similar way during both perturbations periods, and aligns with the apparent angle of the magnetic field in between. The above observations link the polarisation properties to the auroral activity via the clear correlation between the variations of the polarisation properties and the presence of auroras in the line of sight. However this does not necessarily mean that the auroral light is polarised at the source.

Observations in the blue line lead to similar conclusions (Fig. 2, bottom left). Changes in the intensity are correlated with the electron density variations recorded with EISCAT. Furthermore, they are concomitant with high magnetic variability (up to $2 \mathrm{nT} / \mathrm{s}$ around 21:30 UT). These links show again an ionospheric contribution, confirming that the variations in intensity are due to upper atmospheric phenomena. This is the first record of a polarised ionospheric emission, since SPP could only observe the thermosphere through the red line. The DoLP evolves around $2 \%$ during the whole observation period, with variations anti-correlated to the intensity between 10 and 25 min after the start of the record. The AoLP is relatively stable during the first $10 \mathrm{~min}$ (resp. the last $25 \mathrm{~min}$ ), and slightly above (resp. below) the apparent angle of the magnetic field. The shift between these two average positions occurs as the auroral activity peaks.

Finally, the last record in the purple line (Fig. 2, bottom right) shows an intensity that also varies with the electron density, again confirming a contribution from the ionosphere.
The link with magnetic activity is however less clear than for the blue and green line. The DoLP reaches values as high as $5 \%$, and is anti-correlated with the intensity (see for instance the remarkable sharp change about $17 \mathrm{~min}$ after the start of the record). The AoLP starts almost aligned with the magnetic field lines, and then slowly turns away from this position over the observation time-span. It ends up approximately $25^{\circ}$ away from both the field lines orientation and the expected angle for a possible light pollution.

Variations in intensity seem often related to changes in the DoLP. As an example, an anti-correlation is particularly clear for the purple line in Figure 2, and it can also be found at some times with observations in the blue and red lines. If it does not show up in the green line for the case shown in Figure 2, this anti-correlation has been observed in several occurrences in all four wavelengths, and was first put to the fore by Lilensten et al. (2013) in the red.

Similar observations were conducted the day after (March 8, case E), when the magnetic activity was low and a thick layer of clouds was hiding the stars. Figure 3 shows the results obtained for the green line (it is representative of the other lines). As for the above clear sky observations, the electron density measured with EISCAT at $110 \mathrm{~km}$ height and the magnetic field variations are superimposed. Variations in intensity and DoLP are correlated, but cannot be linked to the magnetic and auroral activity. It is different from a cloudless observation where the DoLP and intensity often (but not always) show anti-correlation (Fig. 2). The DoLP values are comparable with what is seen during an aurora (between $0.5 \%$ and $3 \%$ ). The AoLP is constant throughout the observation period, and aligned neither to the magnetic field lines nor to the polarisation expected for Rayleigh scattering of Skibotn lights. We interpret these bursts in intensity and DoLP as being due to changing cloud conditions.

Several features can be emphasized at this point, common to all observations. First, during more than $3 \mathrm{~h}$ almost continuously, we have recorded significant DoLP changes under auroral conditions. With clear sky, all four wavelengths are polarised with a minimum DoLP of $0.5 \%$ in the red line. For the three others, a DoLP over $1 \%$ is maintained over 30 min of observation, with a maximum of $5 \%$ in the purple line. During auroral events, variations in intensity, DoLP and AoLP are correlated to magnetic activity and electron density at the emission, confirming a contribution of the upper atmosphere in the polarisation. As all four wavelengths are polarised, this concerns both the thermosphere (red and green line) and the ionosphere (blue and purple line). Variations in the DoLP and AoLP occur during auroral events. Such changes are linked to the local magnetic activity. This is particularly visible during the first and last $10 \mathrm{~min}$ of the green line record. The AoLP is well defined for the green, blue and purple observation. If slow variations in the AoLP lead to departure by more than $\approx 20^{\circ}$ from the apparent angle of the magnetic field lines for the blue and purple observations, some very dynamic fluctuations, as large as $\approx 70^{\circ}$, are seen during two events on the green record. These variations can not be explained by variations in the magnetic field orientation alone. It may be due to the apparitions of electric field and small scale currents in the upper atmosphere during active times. During this clear-sky observation in the green, we observed twice the same behavior. This is remarkable and could suggest the same phenomenon happened twice. 


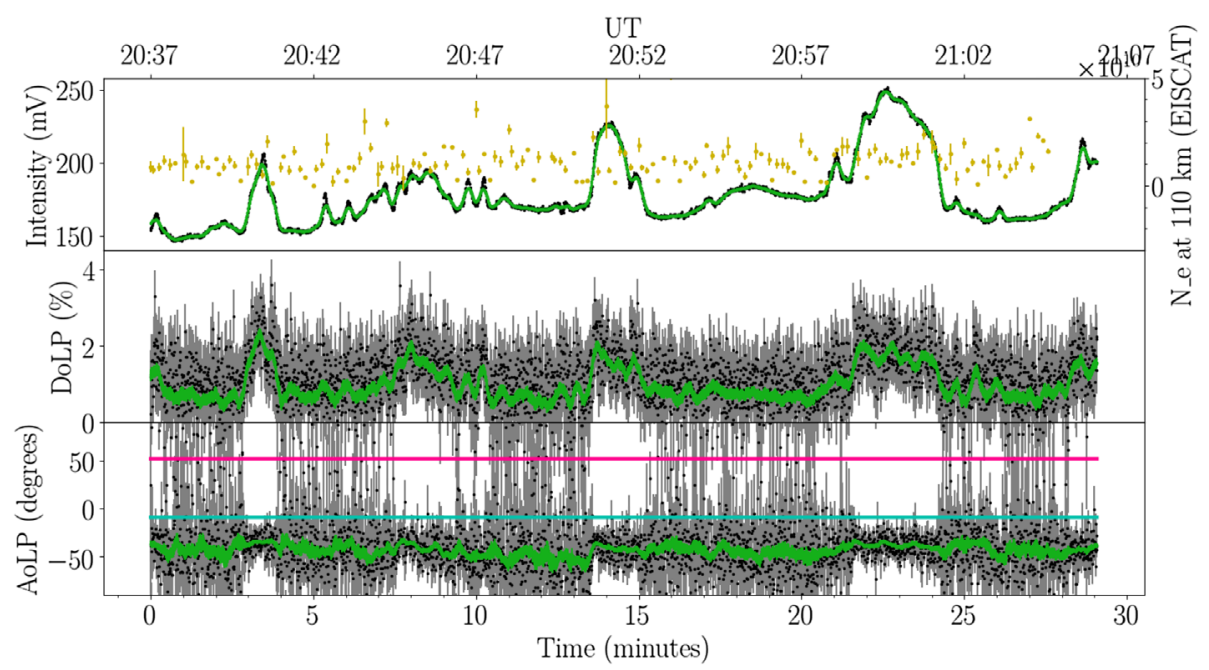

Fig. 3. Green line observation near Skibotn on March 8, 2019, during a cloudy night with low magnetic activity. In black are shown the raw data along with the associated error bars in grey, and in green the smoothed data over $15 \mathrm{~s}$. From top to bottom: the observed intensity (in $\mathrm{mV}$ ), the DoLP (in \%) and the AoLP (in degrees). Electron density at the point of emission is shown in yellow on top of the intensity. The rate of change of the horizontal component of the magnetic field in Troms $\emptyset$ is given in yellow on top of the DoLP. We also show, superimposed with the AoLP, the apparent angle of the magnetic field lines (in cyan) and the expected AoLP for scattered light for a artificial source point in Skibotn (in pink).

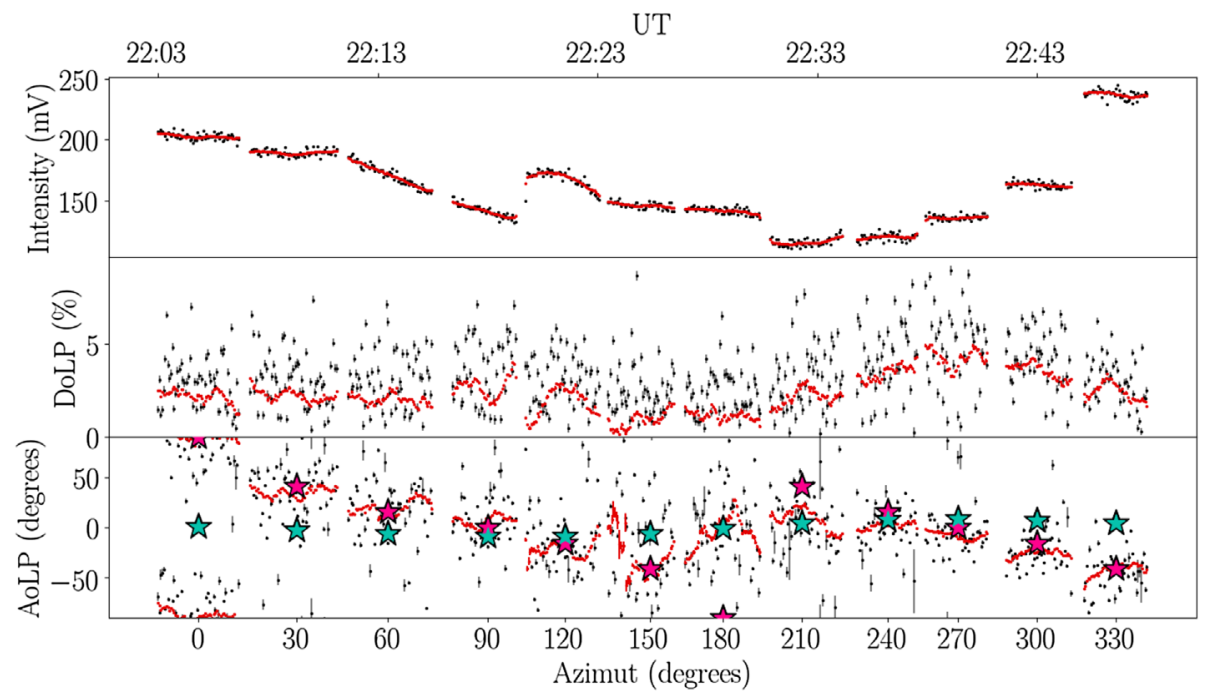

Fig. 4. Red line observation in Ny Alesund on March 3, 2019. We observe at a fixed elevation of $30^{\circ}$, and perform 12 measures at different azimuths (North is 0, and we turn clockwise to the East by steps of $30^{\circ}$ ). In black are shown the raw data along with the associated error bars in grey, and in red the smoothed data over $15 \mathrm{~s}$. From top to bottom: the observed intensity (in mV), the DoLP (in \%) and the AoLP (in degrees). We also show, superimposed with the AoLP, the apparent angle of the magnetic field lines (cyan stars) and the expected AoLP for scattered light for a artificial source point in Ny Alesund, North of the instrument (pink stars).

Finally, the expected angle of polarisation for a point source located in Skibotn does not match the observed AoLP in any of the above observations, even in the presence of clouds. We note that the instrument points the same fixed point in the sky during the entire observation. Since the modeled magnetic field is constant throughout the time period and the direction of the nearest village does not move, the apparent angle of the magnetic field lines and the expected angle for Rayleigh scattering do not change over the whole observation period. Here, both angles are separated by $60^{\circ}$, which is enough to differentiate them.
However, it is not always the case, and these angles are sometimes too close to be properly distinguished with our instrument (under $10^{\circ}$ of separation). We can see this with the almucantar scans performed in Figures 4 and 5: the two angles intersect twice during a whole rotation of the instrument. The existence of an other source of light that, once Rayleigh scattered, would carry an AoLP ambiguous with the apparent angle of the magnetic field lines, can not be neglected. Such a configuration is however not trivial to clearly define, in comparison with that of the nearest village (Skibotn) used in Figures 4 and 5. 

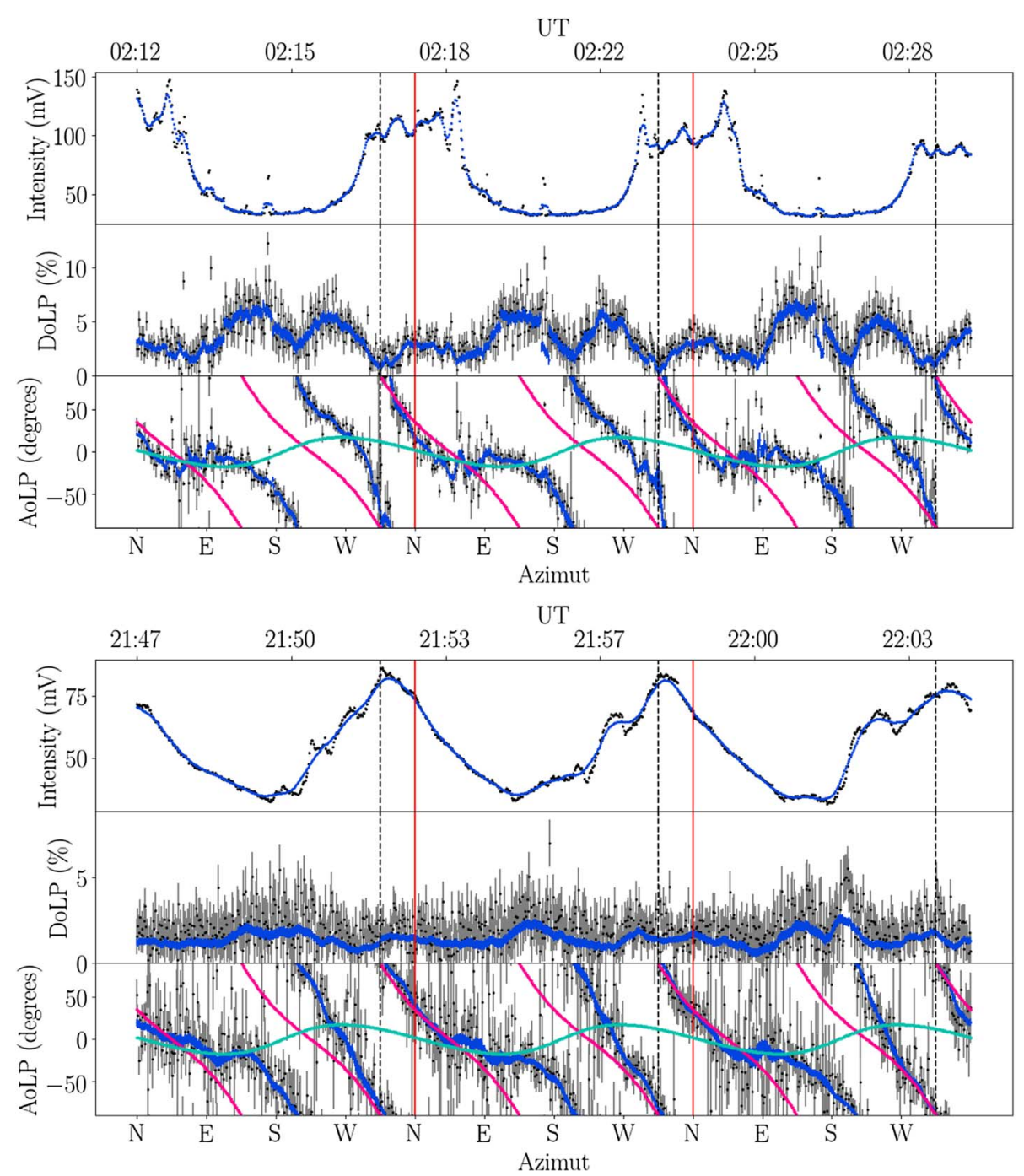

Fig. 5. Blue line observation in Skibotn on March 8, 2019 (top, clear sky) and March 9, 2019 (bottom, cloudy conditions), corresponding to cases $\mathrm{G}$ and $\mathrm{H}$. We observe at a fixed elevation of $45^{\circ}$, and rotate the instrument (fixed on the observatory dome) clockwise starting North, following the same almucantar. Each passage to the North is marked by a vertical red line, and the direction of Skibotn is indicated by the dotted vertical line. In black are the raw data along with the associated error bars in grey, and in blue the smoothed data over $15 \mathrm{~s}$. From top to bottom: the observed intensity (in $\mathrm{mV}$ ), the DoLP (in \%) and the AoLP (in degrees). We also show, superimposed with the AoLP, the apparent angle of the magnetic field lines (cyan) and the expected AoLP for scattered light for a artificial source point in Skibotn (pink).

One can think here about reflections on the snow or bright auroras outside the line of sight, as discussed in Section 3.3. In order to validate the previous observations, we examine below other possible sources of polarisation.

\subsection{The polarisation as an effect of scattering of a secondary source}

A possible cause of polarisation is the contribution from nearby village lights scattered by the atmosphere in the line of sight. Barthélémy et al. (2011) considered this source based on a bibliographic study, and concluded that this effect was negligible. Therefore, it was put aside in later studies related to SPP (e.g. Lilensten et al., 2016). However, we show below that Rayleigh scattered light most likely imprints our observations, and that one must consider the effect from some indirect source(s) of light.
Figure 4 shows an observation of the red line with Petit Cru from Ny Alesund on March 3, 2019 in clear sky with faint auroras visible North-West on the horizon. Twelve measurements of a few minutes each are taken at a fixed elevation of $30^{\circ}$, varying the azimuths, starting North (azimuth $0^{\circ}$ ), and turning clockwise by steps of $30^{\circ}$ towards East. The whole observation takes about $45 \mathrm{~min}$, during which the auroral activity was low and the sky was clear, such that recorded variations are likely related to the direction of observation and not to the time delay. The intensity is almost twice as large to the North than at its minimum to the South-West. This behavior corresponds to the azimuth both of the auroras and of the main village lamps. Over the entire rotation, the DoLP evolves around $1 \%$ and $2 \%$, with a maximum up to $5 \%$ to the West. The AoLP starts at $90^{\circ}$ and turns $180^{\circ}$ over the whole rotation of the instrument. It turns continuously a each step, except between azimuth 150 and 


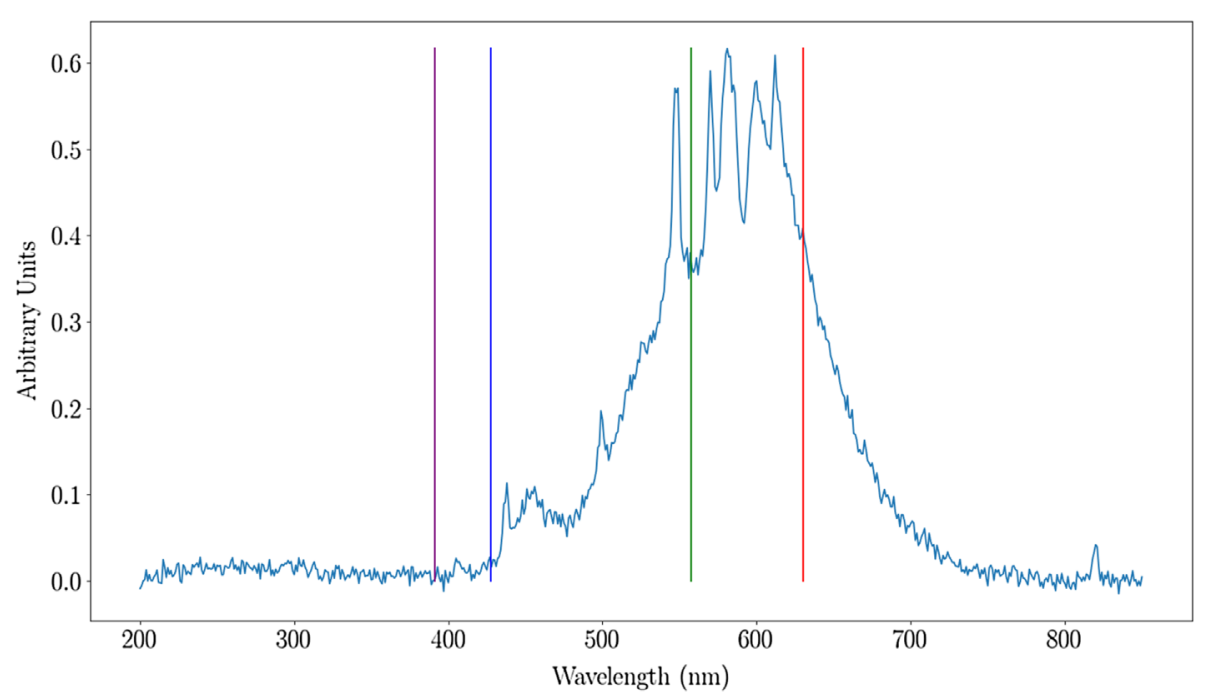

Fig. 6. Average lamp spectral emission in Skibotn. The average of 7 spectra from different lamps is shown (in arbitrary units), as well as the position of the four wavelength observed by Petit Cru. Each individual spectrum was not calibrated in intensity. The lamps emission is negligible below $400 \mathrm{~nm}$, leaving the auroral purple line undisturbed.

240 where it is less well defined and seems to stay constant around $0^{\circ}$. Contrarily to the apparent angle of magnetic field line, the expected AoLP for a source point Northward (corresponding to Ny Alesund lamps) fits the observed AoLP except to the South. The observed AoLP generally matches the expectations for light pollution, and does not coincide with the magnetic field orientation. From this, there is no doubt that the polarisation observed is due to the lamps from Ny Alesund. Only when the village is in our back (pointing southward) can the polarisation be due to another source. However, with such a low DoLP $(<1 \%)$ and a short observation time with SPP, any conclusion remains uncertain.

Figure 5 shows two observations of the blue line taken two days apart in Skibotn, one with a clear sky and auroral activity (case G), the other with a thick cloud cover (case H). Three rotations were performed following a same almucantar. With Petit Cru attached to the observatory dome, we turn at a constant rate (one rotation takes $2^{\prime} 25^{\prime \prime}$ ), with a fixed elevation of $45^{\circ}$. In both cases $\mathrm{G}$ and $\mathrm{H}$ the intensities are doubled around the Northern direction (the direction of the most intense auroras in case $\mathrm{G}$, and of Skibotn in case $\mathrm{H}$ ). However, the shapes of the time-series differ between the two observations. The DoLP in the presence of auroras shows three local maxima per rotation, around $5 \%$ of polarisation (against $1 \%$ for the minima). Instead in the presence of clouds, variations are less clear, and the DoLP is weaker $(<2.5 \%)$.

Nevertheless, the AoLP behaves in a similar manner in both cases. During one almucantar scan, the angle of polarisation performs one entire rotation. The two jumps from $-90^{\circ}$ to $90^{\circ}$ is a graphical artifact (because the direction of polarisation is the same modulo $180^{\circ}$ ). At these latitudes, such a behaviour is expected for polarisation coming from a Rayleigh scattered source. The apparent angle of the magnetic field lines encompasses a much more reduced range of orientations. Nonetheless, it appears that a source located on the ground at Skibotn does not match the observed AoLP over the entire almucantar scan in both cases. The prediction for an artificial source and the observations are off by $50^{\circ}$ during half of the rotation and does not explain the plateau in the observed AoLP seen to the North-East.

If none of the models predicts convincingly the recorded AoLP, the behaviour of the Rayleigh scattered source is much closer than that of the apparent angle of the magnetic field lines. Furthermore, if the polarisation behaves the same way in a clear and cloudy sky, it can not be expected to come from the upper atmosphere. It is plausible that the simple model of point source emission is not complete enough. In this case, a more accurate model might help us discriminate the contribution of light pollution on the polarisation records.

Moreover, another issue deserves to be emphasized. In order to assess the influence of the village lights on our polarisation measurements, we show a spectral measurement of the village lamps (Fig. 6). It gives an idea of the light pollution spectrum in the observation area. Rayleigh scattering is an elastic process, so that the spectral shape does not change after emission. We observe that the minimum wavelength from the village lamps is above $400 \mathrm{~nm}$, thus leaving the $391 \mathrm{~nm}$ line unpolluted. The measurement is not radiometrically calibrated, but there should be no Rayleigh scattered purple light from Skibotn. Furthermore, Skibotn is a small village with few (if any) car traffic at night. The street lamps remain on all night, with absolutely no variation. Therefore, the dynamics revealed in Section 3.1 in the polarisation measurement cannot be attributed to this polluting source.

We show that our polarisation measurements can be and are affected by artificial sources. The DoLP observed in the two cases $\mathrm{G}$ and $\mathrm{H}$ is of the same magnitude as observed in the Section 3.1 (i.e. a few percent). Contrarily to what was previously though (e.g. Lilensten et al., 2016), scattering can not be neglected. Recently, Barthélémy et al. (2019) claimed to have observed the blue line polarisation with a dedicated instrument at the same field station in Skibotn. Their instrument was pointing straight above Skibotn. Moreover, its data processing was based on the assumption that the green line is not polarised, in order to self-calibrate the instrument. We have seen that this 


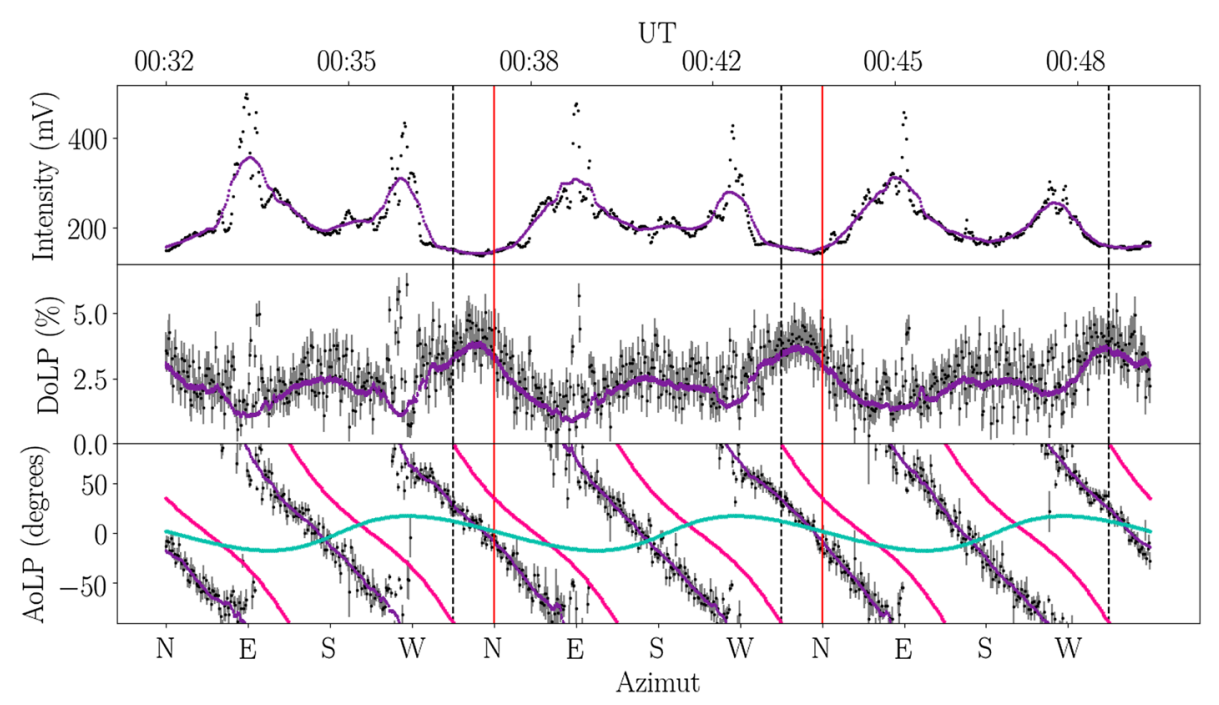

Fig. 7. Purple line observation in Skibotn on March 7, 2019. We observe at a fixed elevation of $45^{\circ}$, and rotate the instrument (fixed on the observatory dome) clockwise starting North, following the same almucantar. Each passage to the North is marked by a vertical red line, and the direction of Skibotn is indicated by the dotted vertical line. In black are the raw data along with the associated error bars in grey, and in blue the smoothed data over $15 \mathrm{~s}$. From top to bottom: the observed intensity (in mV), the DoLP (in \%) and the AoLP (in degrees). We also show, superimposed with the AoLP, the apparent angle of the magnetic field lines (cyan) and the expected AoLP for scattered light for a artificial source point in Skibotn (pink).

last assumption is not valid (whatever the polarisation source). From the present study, we can now insure that the interpretation of the observations by Barthélémy et al. (2019) should be revisited. It is very likely that their detected polarisation was to some extent driven by scattering from the village lamps.

The effect of the artificial sources is complex and is not entirely predicted by our model. Still, the Skibotn site does not seem to be affected for every observation directions and all observations can not be explained with artificial sources polarisation via scattering. Moreover, the purple line is exempt of any artificial contribution from village lights and the dynamics of the polarisation observations cannot be attributed to Skibotn light pollution.

\subsection{The polarisation as an effect of the scattering of a geophysical signal}

From the last section we conclude that if light pollution cannot be neglected easily, all behaviours cannot be explained by scattering from artificial source alone. Figure 7 shows a rotation in purple with again a different behaviour. The intensity shows two peaks towards the East and West, where an auroral arc was present at the time of the observation. The DoLP is anticorrelated with the intensity between $1 \%$ at the minima and up to $4 \%$ at the maxima. The AoLP varies regularly, performing a full rotation of $360^{\circ}$ over one almucantar scan. The expected AoLP for a point source in Skibotn is always turned $30^{\circ}$ away from the expected AoLP due to a source in Skibotn. To correct this effect, we have to put the artificial source $65^{\circ}$ of Skibotn, at an azimuth of $-110^{\circ}$. The observed AoLP does not seem to be affected when crossing in the aurora.

Since the Skibotn lamp spectra have no purple emission (see above), and assuming that the polarised light does not come directly from the aurora, and that Skibotn does not interfere with the measurements, then another source is required. A possibility is that an aurora occurring outside of the line of sight is scattered in the instrument beam-line by the lower atmosphere. If the scattered intensity is high enough, this would induce a measured polarisation linked to the magnetic activity. Auroras are most often dynamic and not comparable to localized sources, and we cannot today predict their effect on the polarisation measurements if scattered in the lower atmosphere. Barthélémy et al. (2011) considered this possibility but concluded that scattered light contribution from a diffuse aurora was negligible. Their modeling was based on the Rayleigh scattering equations, applied to an all sky camera image during a large diffuse aurora. A full description of the effect of an extended and dynamical auroral source out of the field of view requests however a much more complex approach taking into account light transport through a modeled atmosphere. This is out of scope of the present study, but should be thoroughly considered in the future.

\subsection{The polarisation as an effect of Faraday rotation}

In search of phenomena interacting with the auroral light polarisation, we look into the Faraday effect and how it affects the AoLP through the ionosphere (Hatanaka, 1956; Daniels \& Bauer, 1959). Electromagnetic waves propagating in any ionized medium undergo a rotation of polarised components of the electric field, known as Faraday effect. It is an interaction between the wave and the magnetic field of the medium. Linearly polarised electromagnetic waves crossing the ionosphere are also subject to Faraday effect. Due to the presence of free electrons (heavier ions are considered immobile), the ionosphere can support only two modes of circular polarisation right and left (only the geomagnetic field is taken into account; the magnetic field of the wave is neglected in the non-relativist limit). At the edge of the ionosphere, the electromagnetic wave 
decomposes into two circular polarised components. Each one propagates with a velocity slightly different from the other so that at the end, they recombine giving a linearly polarised wave but whose polarisation plane have undergone a rotation angle $\Omega$ relative to the polarisation plane of the incident wave. Faraday rotation angle (in degree) can be estimated (Jehle et al., 2009) as

$$
\Omega=\frac{2.36510^{4} \lambda^{2}}{C^{2}} B_{\|} \int_{0}^{h} N_{e} \mathrm{~d} h=\frac{2.36510^{4}}{f^{2}} V T E C \frac{1}{\cos \gamma} B_{\|},
$$

where $B_{\|}$(in Teslas) is the mean geomagnetic field in the line of sight of the instrument (inside the crossed part of the ionosphere), $c$ is the speed of light, $\lambda$ is the wavelength, $f$ (in $\mathrm{GHz}$ ) is the wave frequency, VTEC (in TECU so that 1 TECU = $10^{16}$ electron $/ \mathrm{m}^{2}$ ) is the vertical total electron content and $1 / \cos \gamma$ is the factor which converts the VTEC to the TEC along the wave propagation direction.

If we take into account that our measurements concern waves in the visible range of the electromagnetic spectrum $\left(f \in[3.8,7.9] 10^{5} \mathrm{GHz}\right)$, a TEC of the order of $10^{3}$ TECU, and $B_{\|}$of the order of $50 \mu \mathrm{T}$, a simple numerical estimate indicate that $\Omega$ is less than one tenth of a degree.

\section{Discussion}

Our results show a large variety of behaviours of the recorded polarisation in a natural environment, and raise many questions. All four wavelengths observed by our instrument are polarised. Is this characteristic of auroral emissions or common to all wavelength? We clearly link the polarisation behaviour to the auroral activity. However, is the polarisation coming from the emission itself or is the light polarised along the way?

\subsection{How is the green light polarised?}

From Bommier et al. (2011), we expect the green auroral line to be unpolarised at the emission. However, the green line is polarised when entering our instrument. Two options are plausible. Either, there is a way to polarise the green line at the emission, which is not predicted by our current knowledge of the atomic oxygen model. In that case, we can use it to study the upper atmosphere, like the other auroral lines. Alternatively, the polarisation comes from somewhere else and the only way we can think of is through scattering.

As we have shown, the closest village can not by itself explain all polarisation behaviour (in particular for the purple line). An other source is needed, like an aurora outside the line of sight, or something else altogether. Anyhow, if the polarisation comes from the lower atmosphere, one of the four lines could be used to monitor its contribution. Indeed, with a proper scattering model linking the polarisation properties of all four lines, one could imagine characterizing and subtracting the lower atmosphere contribution. This could allow observations in more places, without such strong criteria about light pollution.

\section{2 lonospheric applications of polarisation}

In cases were lower atmosphere contributions were to be negligible (or modeled), the observed polarisation is due to upper atmospheric phenomena. Our first guess was the alignment of the AoLP and the magnetic field lines. During periods of magnetic auroral activity, the magnetic field angle can vary on the order of a few percent. Even if the relation between the variations in strength and the apparent direction on the instrument is not linear, it is hard to explain such large and long variations of the AoLP. However, the flow of electrons exciting the upper atmosphere does not have to always be aligned on the magnetic field. For example an electric field could be strong enough to steer them around. In that case, the polarisation is not expected to follow magnetic field lines, but can vary with the ionospheric currents. This possibility will be explored in the future.

\subsection{The tropospheric applications of polarisation}

Another possibility could explain at least part of our observations: the polarisation could be affected when crossing the lower atmosphere. Several mechanisms may compete. The first one is the presence of aerosols. An aerosol is a suspension of fine solid particles or liquid droplets in the air. They are measured in particular with Lidars (Ferrero et al., 2019). Lidars measure backscatter and extinction coefficients but also the polarisation of light, the later providing an information on the shape of the particles.

There is a Lidar in Ny Alesund owned by the Alfred Wegener Institute Helmholtz, center for Polar and Marine Research/ AWI, Department of Atmospheric Circulation. We tried to compare its measurements with our polarisation measurements. Unfortunately, we could only find very sparse times with coordinated operation, not enough to conclude about this effect. The operator of the Lidar, Christoph Ritter (personal communication), mentioned to us: "In total the aerosol load in the Arctic is quite low and it is clearer in autumns and more polluted in spring (Zielinski et al., 2020). Hence if aerosol were the reason I would expect that you see in spring on more days more erratic polarisation than in autumn." This is no proof that the aerosols can be excluded. In particular, should they play a role, this polarisation technique would open a new way to measure them. Indeed, there is no way to measure their presence and characteristics at night over a large area with a passive experiment. In our case, we use the nightglow (which exists at all latitudes) as a projector and measure its deformation to induce the atmospheric properties. Therefore, it is of great importance in the frame of air pollution studies to keep exploring this way.

Christoph Ritter also mentions: "If phenomena like this were to disturb your measurements I would speculate that this would give an erratic deviation to the polarisation you are expecting and not a constant offset". That is indeed true since the aerosols move permanently in any direction, changing randomly the polarisation angle, and thus providing a strong argument against their implication. Finally, their presence lasts for hours and can hardly explain our rapid variations (Zielinski et al., 2020).

Apart from aerosol, we sometimes see in the Arctic spurious ice crystals or even thin cirrus clouds through which the aurora are still visible (Cai et al., 2011). They are optically thin (low backscatter and extinction) but they do change the polarisation. The time scale may be of the order of a few seconds. In the few times when we could compare our measurements with that of the Lidar, we saw however no trace of such ice crystals nor 
cirrus clouds. Again, this does not fully rule out these assumptions, which must be explored further, but it gives more weight on a polarisation originating directly at the light emission in the upper atmosphere (if not Rayleigh diffused).

\section{Conclusion}

Observations with the new instrument uncovered a lot of unexpected results, making the interpretations more complex and subtle compared to what was seen previously with SPP or by Barthélémy et al. (2019). Measures show very different behaviours of the polarisation through time, and for the several wavelengths. The anti-correlation between intensity and DoLP is still visible in most of the records. Several sources can induce polarisation in our signal, that are not always easy to disentangle. There is still the possibility of a direct polarisation from the aurora, but as well from scattering of village lights in the lower atmosphere, or scattering of auroral lights themselves. The difficulty lies in the fact that the physics behind the nightglow polarisation is not perfectly understood, and modelling the expected polarisation is still at a premature stage. The ionosphere is a dynamical environment and we are not sure what to expect from direct polarisation. To discriminate between these three sources, we are working on multi instrumental observations, using the EISCAT antenna, Lidar measurements of the aerosols and a radiative transfer model of the troposphere contribution using all-sky camera data. We also develop multi-channels instruments with an integrated wide field camera. In the future, polarisation could become an observable of the upper and lower atmosphere. On the one hand it could help to observe magnetic field and/or currents in the ionosphere, or alternatively to provide information on aerosols or light pollution in the troposphere.

Acknowledgements. This work was partly funded by the French polar institute IPEV under program POLARLIS 3, number 1026. It was also funded by the Prematuration CNRS program and by the Maturation program CM180023, project PTCU Number $180018 \mathrm{M}$ from the SATT company Linksium. EISCAT is an international association supported by research organisations in China (CRIRP), Finland (SA), Japan (NIPR and ISEE), Norway (NFR), Sweden (VR), and the United Kingdom (UKRI). This work was supported by the Programme National PNST of CNRS/INSU co-funded by CNES and CEA. The polarimeter is patented by the CNRS and valorised by Linksium (international patent number 1873378). We also want to thank the University of Oslo for providing the all-sky images through there webpage.

The editor thanks Frans Snik and Fred Sigernes for their assistance in evaluating this paper.

\section{References}

Auriol F, Léon J-F, Balois J-Y, Verwaerde C, François P, et al. 2008. Multidirectional visible and shortwave infrared polarimeter for atmospheric aerosol and cloud observation: OSIRIS (Observing System Including PolaRisation in the Solar Infrared Spectrum).
In: Larar AM, Lynch MJ, Suzuki M (Eds.) Multispectral, hyperspectral, and ultraspectral remote sensing technology, techniques, and applications II, Vol 7149, International Society for Optics and Photonics, SPIE. pp. 235-246. https://doi.org/ 10.1117/12.806421.

Baron M. 1986. EISCAT Progress 1983-1985. J Atmos Terr Phys 48 (9-10): 767-772. https://doi.org/10.1016/0021-9169(86)90050-4.

Barthélémy M, Lilensten J, Pitout F, Simon Wedlund C, Thissen R, et al. 2011. Polarisation in the auroral red line during coordinated EISCAT Svalbard Radar/optical experiments. Ann Geophys 29(6): 1101-1112. https://doi.org/10.5194/angeo-29-1101-2011.

Barthélémy M, Lamy H, Vialatte A, Johnsen MG, Cessateur G, Zaourar N. 2019. Measurement of the polarisation in the auroral $\mathrm{N}_{2}^{+} 427.8 \mathrm{~nm}$ band. J Space Weather Space Clim 9: A26. https://doi.org/10.1051/swsc/2019024.

Bohren CF, Huffman DR. 2008. Absorption and scattering of light by small particles (e-book). John Wiley \& Sons. ISBN 978-3-52761816-3.

Bommier V, Sahal-Bréchot S, Dubau J, Cornille M. 2011. The theoretical impact polarization of the O I $6300 \AA$ red line of earth aurorae. Ann Geophys 29(1): 71-79. https://doi.org/10.5194/ angeo-29-71-2011.

Cai H, Zhou R, Fu Y. 2011. Cloud-aerosol lidar with orthogonal polarisation detection of aerosol optical properties after a crop burning case. Clim Environ Res 16: 469-478.

Chamberlain J. 1959. On the polarisation of the oxygen red line in aurorae. Planet Space Sci 2(1): 73-75. https://doi.org/10.1016/ 0032-0633(59)90063-7.

Daniels FB, Bauer SJ. 1959. The ionispheric Faraday effect and its applications. J Franklin Ins 267(3): 187-200. https://doi.org/ 10.1016/0016-0032(59)90133-4.

Duncan R. 1959. polarisation of the red oxygen auroral line. Planet Space Sci 1(2): 112-114. https://doi.org/10.1016/0032-0633(59) 90006-6.

Ferrero L, Ritter C, Cappelletti D, Moroni B, Monik G, et al. 2019. Aerosol optical properties in the Arctic: The role of aerosol chemistry and dust composition in a closure experiment between Lidar and tethered balloon vertical profiles. Sci Total Environ 686: 452-467. https://doi.org/10.1016/j.scitotenv.2019.05.399.

Finlay CC, Olsen N, Kotsiaros S, Gillet N, Tøffner-Clausen L. 2016. Recent geomagnetic secular variation from Swarm and ground observatories as estimated in the CHAOS-6 geomagnetic field model. Earth Planets Space 68(1): 112. https://doi.org/10.1186/ s40623-016-0486-1.

Finlay C, Lesur V, Thébault E, Vervelidou F, Morschhauser A, Shore R. 2017. Challenges handling magnetospheric and ionospheric signals in internal geomagnetic field modelling. Space Sci Rev 206: 157-189. https://doi.org/10.1007/s11214-016-0285-9.

Harang L. 1933. Eine Untersuchung der Polarisation des Nordlichtes. Z Geophysik 9: 162-164.

Harang O. 1960. An investigation of the state of polarisation of the red and green 3-lines in polar aurorae. Technical Report. Norway, Oslo University.

Hatanaka T. 1956. The Faraday effect in the earth's ionosphere with special reference to polarisation measurements of the solar radio emission. Publ Astron Soc Japan 8: 73.

Jehle M, Rüegg M, Zuberbühler L, Small D, Meier E. 2009. Measurement of ionospheric Faraday rotation in simulated and real spaceborne SAR data. IEEE Trans Geoscie Remote Sens 47(5): 1512-1523. https://doi.org/10.1109/tgrs.2008.2004710.

Johnsen MG. 2013. Real-time determination and monitoring of the auroral electrojet boundaries. J. Space Weather Space Clim. 3: A28. https://doi.org/10.1051/swsc/2013050. 
Lilensten J, Simon C, Barthélémy M, Moen J, Thissen R, Lorentzen DA. 2006. Considering the polarisation of the oxygen thermospheric red line for space weather studies: polarisation of oxygen thermospheric red line. Space Weather 4(11): S11002. https://doi. org/10.1029/2006SW000228.

Lilensten J, Moen J, Barthélemy M, Thissen R, Simon C, Lorentzen DA, Dutuit O, Amblard PO, Sigernes F. 2008. Polarization in aurorae: A new dimension for space environments studies. Geophys Res Lett 35(8): L08804. https://doi.org/10.1029/2007GL033006.

Lilensten J, Barthélémy M, Amblard P-O, Lamy H, Wedlund CS, Bommier V, et al. 2013. The thermospheric auroral red line polarisation: confirmation of detection and first quantitative analysis. $J$ Space Weather Space Clim 3: A01. https://doi.org/10.1051/swsc/2012023.

Lilensten J, Bommier V, Barthélemy M, Lamy H, Bernard D, Moen JI, Johnsen MG, Løvhaug UP, Pitout F. 2015. The auroral red line polarisation: modelling and measurements. $J$ Space Weather Space Clim 5: A26. https://doi.org/10.1051/swsc/2015027.

Lilensten J, Barthélemy M, Besson G, Lamy H, Johnsen MG, Moen J. 2016. The thermospheric auroral red line angle of linear polarization. J Geophys Res (Space Phys) 121(7): 7125-7134. https://doi.org/10.1002/2016JA022941.

Meek JH. 1953. Correlation of magnetic, auroral, and ionospheric variations at Saskatoon. J Geophys Res (1896-1977) 58(4): 445456. https://doi.org/10.1029/JZ058i004p00445.

Picinbono B. 1993. Random signals and systems. Prentice Hall. ISBN 978-0-13-752270-5.

Rice SO. 1945. Mathematical analysis of random noise. Part III: Statistical porperties of random noise currents. Bell System Tech J Bell Labs. 24: 41-156. https://doi.org/10.1002/j.1538-7305.1945.tb00453.x.

Sparks WB, Axon DJ. 1999. Panoramic polarimetry data analysis. Publ Astron Soc Pac 111(764): 1298-1315. https://doi.org/10. 1086/316434.

Thébault E, Finlay CC, Beggan CD, Alken P, Aubert J, et al. 2015. International geomagnetic reference field: the 12th generation. Earth Planets Space 67: 79. https://doi.org/10.1186/s40623-015-0228-9.

Van Tree H. 1968. Detection, estimation and modulation theory, Vol I. Wiley. ISBN 978-0-470-54296-5.

Zielinski T, Bolzacchini E, Cataldi M, Ferrero L, Graß1 S, et al. 2020. Study of chemical and optical properties of biomass burning aerosols during long-range transport events toward the arctic in summer 2017. Atmosphere 11: 84. https://doi.org/10.3390/atmos11010084.

\section{Appendix A}

\section{Lock-in method and computation of measurements error}

Petit Cru selects photons at a particular wavelength (thanks to an interferential filter), a fraction of which are supposed to be linearly polarized. To measure the polarization parameters (DoLP and AoLP), Petit Cru further selects photons through a rotating polarizing filter. The rotation angle is defined to be $\theta_{t} / 2$ as a function of time $t$. The device has one full rotation in $T_{0}$ seconds, and the motion is stable from one rotation to another, $\theta_{t+k T_{0}}=\theta_{t}+2 k \pi$. The number of photons going through the whole optical device per unit of time can then be modelled as a Poisson random variable with rate,

$$
\zeta_{t}=\zeta_{0}+\zeta \cos \left(\theta_{t}+\varphi\right)
$$

$\zeta_{0}$ and $\zeta$ count respectively the unpolarized photons and the linearly polarized photons - in units of a number of photons per second. In the following they are assumed to be constant in time to allow the design of an estimation procedure. The photons then enter a photomultiplier that delivers a current varying linearly with the photon rate.

When the rate of a Poisson random variable is large, the Poisson variable can be approximated with a Gaussian variable with same mean and variance (Picinbono, 1993). This allows modeling the output of the photomultiplier as a random Gaussian process $s_{t}$ with mean $\zeta_{t}=\zeta_{0}+\zeta \cos \left(\theta_{t}+\varphi\right)$ and variance $\zeta_{t}=\zeta_{0}+\zeta \cos \left(\theta_{t}+\varphi\right)$. Thus, we can write $s_{t}$ as

$$
s_{t}=\zeta_{t}+\sqrt{\zeta_{t}} w_{t}
$$

where $w_{t}$ is a Gaussian process with zero mean and unit variance. We suppose that the correlation time of $w_{t}$ is much shorter than the period of rotation $T_{0}$ of the device. We thus consider in the sequel that $w_{t}$ is a white Gaussian noise. For the sake of simplicity, we abusively keep the same notations for the parameters of interest $\left(\zeta_{0}, \zeta, \varphi\right)$ from the Poisson model and those of the Gaussian model. Note also that $\zeta_{0}$ not only represents the unpolarized light, but also contains a term representing the dark current of the device. This dark current is regularly monitored and subtracted from the measurements.

The goal of Petit Cru is to deliver the incoming intensity $I_{0}$, the DoLP and the AoLP, which are related to the above parameters via equation (1) as

$$
I_{0}=2 \zeta_{0}, \operatorname{DoLP}=\zeta / \zeta_{0}, \operatorname{AoLP}=-\varphi / 2 .
$$

Therefore, the estimation (or measurement) of the parameters $\zeta_{0}, \zeta, \varphi$ must be extracted from the data collected by Petit $\mathrm{Cru}$.

The signal model (A.2) and the fact that $\theta_{t}$ is measured by Petit Cru leads to the use of synchronous detection as a means of extracting pertinent statistics of the parameters. Synchronous detection allows to access the information that are modulating the sinusoidal waveform $\cos \left(\theta_{t}\right)$. It simply consists in multiplying the signal by the same waveform, and averaging over a period. Since two parameters appears in the model, this action must be performed twice, with two orthogonal sinusoidal waveform. For each rotation $i$, we therefore calculate

$$
\left\{\begin{array}{l}
V_{c, i}=\frac{1}{T_{0}} \int_{0}^{T_{0}} s_{t} \cos 2 \theta_{t} \mathrm{~d} t \\
V_{s, i}=-\frac{1}{T_{0}} \int_{0}^{T_{0}} s_{t} \sin 2 \theta_{t} \mathrm{~d} t . \\
V_{i}=\frac{1}{T_{0}} \int_{0}^{T_{0}} s_{t} \mathrm{~d} t
\end{array}\right.
$$

Easy calculations allows to obtain the statistics of these samples. Since $s_{t}$ is a Gaussian random signal, it is easy to show that $\boldsymbol{v}_{i}=\left(V_{c, i}, V_{s, i}, V_{i}\right)$ is a Gaussian random vector with mean $m$ and covariance matrix $C$ given by:

$$
m=\left(\begin{array}{c}
\frac{\zeta}{2} \cos \varphi \\
\frac{\zeta}{2} \sin \varphi \\
\zeta_{0}
\end{array}\right) \quad \text { and } \quad C=\left(\begin{array}{ccc}
\frac{\zeta_{0}}{2 T_{0}} & 0 & \frac{\zeta}{2} \cos \varphi \\
0 & \frac{\zeta 0}{2 T_{0}} & \frac{\zeta}{2} \sin \varphi \\
\frac{\zeta}{2} \cos \varphi & \frac{\zeta}{2} \sin \varphi & \frac{\zeta}{T_{0}}
\end{array}\right)
$$


Note that $V_{c, i}$ and $V_{s, i}$ are uncorrelated (and thus independent since they are Gaussian). However, $V_{c, i}$ and $V_{s, i}$ are correlated with $V_{i}$. This complicates the optimal estimation of the parameters. We will therefore adopt a simpler strategy assuming (wrongly) that $V_{c, i}$ and $V_{s, i}$ are also decorrelated from $V_{i}$.

The optimal approach then relies on the maximum likelihood paradigm (Van Tree, 1968; Picinbono, 1993). Note that this paradigm for polarimetric data analysis has already been used in the past in other communities, see for example Sparks and Axon (1999). The likelihood of the data is the probability density function (pdf) assumed for the data evaluated at the data, and seen as a function of the parameters. Here, the pdf of the data is supposed to be $g\left(\boldsymbol{r} ; \zeta_{0}, \zeta, \varphi\right)$ a three dimensional Gaussian distribution with mean $m$ and covariance $C$ given above (assumed to be diagonal as discussed). Thus the likelihood of one $\boldsymbol{v}_{i}=\left(V_{c, i}, V_{s, i}, V_{i}\right)$ is $g\left(\boldsymbol{v}_{i} ; \zeta_{0}, \zeta, \varphi\right)$, and the likelihood of $N$ observations is the product of such functions, since we assume the observations to be independent. The maximum likelihood paradigm states that a good estimate of the true parameters should maximize the likelihood viewed as a function of these parameters. The log-likelihood is usually used for the sake of simplicity. For $N$ observations ( $N$ rotations of the polarizer), it writes

$$
\begin{aligned}
\log p=-\sum_{i=1}^{N} & \left\{\frac{T_{0}}{\zeta_{0}}\left(V_{c, i}-\frac{\zeta}{2} \cos \varphi\right)^{2}-\frac{T_{0}}{\zeta_{0}}\left(V_{s, i}-\frac{\zeta}{2} \sin \varphi\right)^{2}\right. \\
& \left.-\frac{T_{0}}{2 \zeta_{0}}\left(V_{i}-\zeta_{0}\right)^{2}\right\}-\frac{3 N}{2} \log \frac{\zeta_{0}}{T_{0}}
\end{aligned}
$$

Taking the gradient of the log-likelihood with respect to the parameters, equating it to zero and solving allows to find the following estimators:

$$
\begin{gathered}
\widehat{\zeta}=\frac{2}{N} \sum_{i}\left(V_{c, i} \cos \widehat{\varphi}+V_{s, i} \sin \widehat{\varphi}\right), \\
\widehat{\varphi}=\arctan \frac{\sum_{i} V_{s, i}}{\sum_{i} V_{c, i}}, \\
\widehat{\zeta_{0}}= \\
\frac{-3+\sqrt{9+\frac{8 T_{0}^{2}}{N} \sum_{i}\left(\left(V_{c, i}-\frac{\hat{\zeta}}{2} \cos \widehat{\varphi}\right)^{2}+\left(V_{s, i}-\frac{\hat{\zeta}}{2} \sin \widehat{\varphi}\right)^{2}+\frac{1}{2} V_{i}^{2}\right)}}{2 T_{0}} .
\end{gathered}
$$

These estimators are coupled. We need to estimate the phase first and then $\zeta$. The full expression for $\zeta_{0}$ is rather complicated. However, a quick look at the orders of magnitude of the different terms allows to write the estimator simply as $\widehat{\zeta_{0}}=\sqrt{N^{-1} \sum_{i} V_{i}^{2}}$. The form of this estimate may look strange at a first glance. It comes from the Gaussian approximation to the Poisson process which implies that $\zeta_{0}$ is the mean as well as the variance. Clearly, a simpler estimator is nothing but the empirical mean of $V_{\mathrm{i}}$. A statistical analysis allows to conclude that both have the same variance. We will thus use

$$
\widehat{\zeta_{0}}=\frac{1}{N} \sum_{i=1}^{N} V_{i} .
$$

Finally, we can easily rewrite the estimators as a function of the empirical mean of the variable $\left(V_{c, i}, V_{s, i}, V_{i}\right)$ that we respectively denote as $\bar{V}_{c}, \bar{V}_{s}, \bar{V}$. We indeed have

$$
\left\{\begin{array}{l}
\widehat{\zeta}=2 \sqrt{\bar{V}_{c}^{2}+\bar{V}_{s}^{2}} \\
\widehat{\varphi}=\arctan \frac{\bar{V}_{s}}{\bar{V}_{c}} \\
\widehat{\zeta_{0}}=\bar{V}
\end{array}\right.
$$

With our assumption the triplet $\bar{V}_{c}, \bar{V}_{s}, \bar{V}$ is a three dimensional Gaussian with parameters

$$
m=\left(\begin{array}{c}
\frac{\zeta}{2} \cos \varphi \\
\frac{\zeta}{2} \sin \varphi \\
\zeta_{0}
\end{array}\right) \quad \text { and } \quad C=\left(\begin{array}{ccc}
\frac{\zeta_{0}}{2 N T_{0}} & 0 & 0 \\
0 & \frac{\zeta_{0}}{2 N T_{0}} & 0 \\
0 & 0 & \frac{\zeta_{0}}{N T_{0}}
\end{array}\right)
$$

Therefore $\widehat{\zeta}_{0}$ is clearly an unbiased estimator (its mean is $\zeta_{0}$ ) and its variance is $\frac{\zeta_{0}}{N T_{0}}$. Furthermore its distribution is Gaussian, allowing to compute its confidence interval easily. For example, an interval of width $2 \sqrt{\frac{\zeta_{0}}{N T_{0}}}$ centered in $\zeta_{0}$ is a $95 \%$ confidence interval.

The estimators $\widehat{\zeta}$ and $\widehat{\varphi}$ are coupled. They can however be studied (Rice, 1945; Picinbono, 1993). Their joint distribution is known explicitly, as well as the marginal distributions. However, the moments are difficult to evaluate. For $\zeta$ it can be shown for example that the two first moments read

$$
\left\{\begin{array}{l}
E[\widehat{\zeta}]=2 \sqrt{\frac{\zeta_{0}}{2 N T_{0}}} \sqrt{\frac{\pi}{2}} L_{\frac{1}{2}}\left(-\frac{\zeta^{2} N T_{0}}{4 \zeta_{0}}\right) \\
E\left[\widehat{\zeta}^{2}\right]=\frac{4 \zeta_{0}}{N T_{0}}+\zeta^{2}
\end{array}\right.
$$

from which we can evaluate the variance. $L_{1 / 2}$ is a Laguerre function. This shows that $\widehat{\zeta}$ is a biased estimator.

However and importantly, it appears that the behavior of these estimators is closely linked to the magnitude of parameter

$$
S / N=\frac{\zeta}{\sqrt{2 \zeta_{0}}} \sqrt{N T_{0}},
$$

which can be interpreted as a signal to noise ratio. It is the ratio of vector $\left(\bar{V}_{c}, \bar{V}_{s}\right)$ mean length over its standard deviations. When $S / N$ is large, $\widehat{\zeta}$ converges to a Gaussian random variable with mean $\zeta$ and variance $2 \zeta_{0} /\left(\mathrm{NT}_{0}\right)$, and $\widehat{\varphi}$ also converges to a Gaussian random variable with mean $\varphi$ and variance

$$
\operatorname{var}[\widehat{\varphi}]=\frac{2 \zeta_{0}}{\zeta^{2} N T_{0}} .
$$

It is commonly accepted that convergence is rapid meaning that $S / N$ is large as soon as it is typically greater than 4 or 5.

The estimated DoLP is then $\widehat{\zeta} / \widehat{\zeta}_{0}$. Assuming that the variance of the estimators $\widehat{\zeta}$ and $\widehat{\zeta_{0}}$ is small compared to their mean, we can approximate the mean and the variance of their ratio. The ratio is unbiased (up to $1 /\left(N T_{0}\right)$ term) and the variance reads

$$
\operatorname{var}\left[\frac{\widehat{\zeta}}{\widehat{\zeta_{0}}}\right] \approx \frac{2}{\zeta_{0} N T_{0}}\left(1+\frac{\zeta^{2}}{2 \zeta_{0}^{2}}\right),
$$

the correction being of order $1 /\left(N T_{0}\right)^{3 / 2}$. 


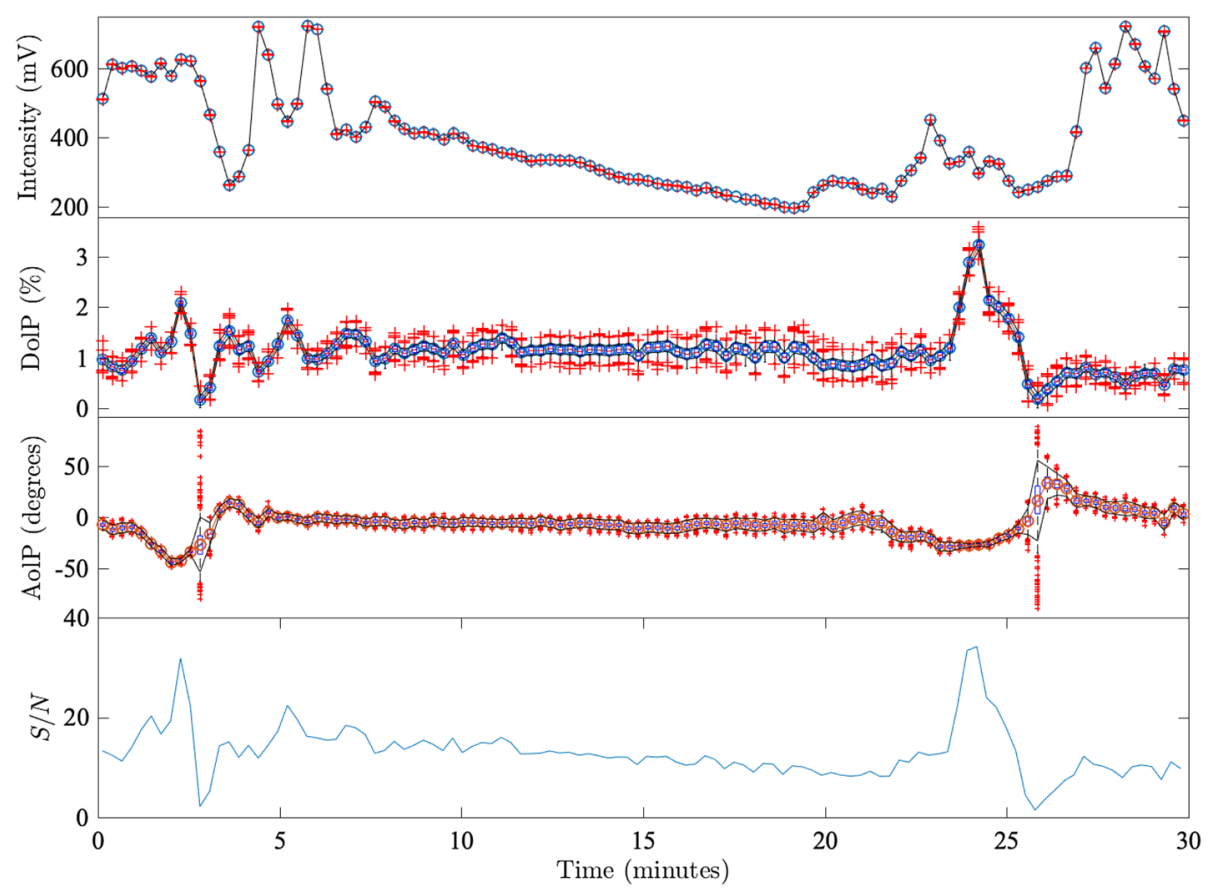

Fig. A.1. Bootstraped analysis of the green line observed on March 7, 2019, as in Figure 2. For the intensity, DoLP and AoLP, we superpose as dotted line the estimated parameters as in Figure 2, to the boxplot (histogram) of the bootstrapped parameters. 1000 realisations have been calculated using a parametric model of Petit Cru. On each realisation, the parameters are reestimated, allowing the statistical analysis displayed. The box contains half of the reestimated parameters (between 25 and 75\% percentiles). Red crosses correspond to outliers as decided by the automatic plotting routine. The parameter $S / N$ completes the plot. As can be observed, low values of $S / N$ lead to poor estimates of the angle, and to a higher bias in the DoLP.

All the useful results presented above rely on the Gaussian assumption, and suppose obviously that the phenomenon studied is stationary over the duration of observation (basically the parameters do not evolve during $N T_{0}$ ). The Gaussian assumption is realistic for the models of the received signals, but is critical when it comes to analyse the estimators. As we mentioned, the requirement is that the critical parameter $S / N=$ $\frac{\zeta}{\sqrt{2 \zeta_{0}}} \sqrt{N T_{0}}$ is large, in practice greater than 4 or 5 . For a given measure, increasing $S / N$ requires to increase $N T_{0}$. This implies that the sky observed is stationary over a longer period of time.

If $S / N$ is small, statistical difficulties arise and the interpretation of the measurement must be done with caution. Furthermore, in this situation, the estimated $\zeta$ is biased. Even if this bias can be estimated and corrected (see e.g. Sparks \& Axon, 1999), we do not do it here, since the bigger problem comes from the phase which is hardly interpretable in this situation.

To illustrate all these points we propose here a bootstrapped analysis of one of the measurement presented in the paper. We chose the green line in Figure 2. By bootstrap, we mean that we estimated all the parameters as done above in equation (A.7) and in the core of the paper, and we used these parameters as inputs in the parametric model of the signals defined by equations (A.1) and (A.2). The demodulation step is then done using (A.4), and the new estimation of the parameters for this surrogate measurement using again (A.7).

From the green line estimated parameters, we have generated 1000 different surrogates of the same signal. We then applied the estimation procedure on these surrogate signals and were then able to perform empirical statistical analysis of the estimation algorithm. In Figure A.1, we plot a synthesis of this bootsrapped analysis. The top plot represent the intensity $I_{0}$, the second one the DoLP, the third plot the AoLP. We have added in the fourth plot the evolution of the critical parameter $S / N$. In the three first plots, we depict the parameters as estimated in Figure 2, superposed with a boxplot synthesising the boostrap analysis. The box contains $50 \%$ (between $25 \%$ and $75 \%$ percentiles) of the realisations, the top and bottom whiskers are the max and the min, whereas the red crosses represent outliers as judged by the automatic plotting procedure. We have the clear confirmation that estimation of the intensity is really easy since the dispersion of the measurement is very small: the boxes are highly concentrated around the mean value. The other important confirmation is the dependence of the quality of the measurement of DoLP and AoLP on parameter $S / N$. When $S / N$ is lower than say 5 , the DoLP is biased and the AoLP very badly estimated. The bias could be corrected. However, AoLP is so badly estimated when $S / N<5$ that the measurement in this situation are hardly interpretable, and should be used with much caution. This is particularly clear when examining the plots in Figure A.1, around time $3 \mathrm{~min}$ and $26 \mathrm{~min}$. At these times, $S / N$ is around 2 and the histogram of AoLP particularly large, making a prediction impossible.

To be more precise we chose two times illustrating at two different $S / N$ the histograms calculated using the surrogates in Figure A.2. At time $21.37 \mathrm{~min}$, parameter $S / N$ is large at 9.4, whereas at time $25.82 \mathrm{~min}, S / N$ is small at 1.54 . We plot for 

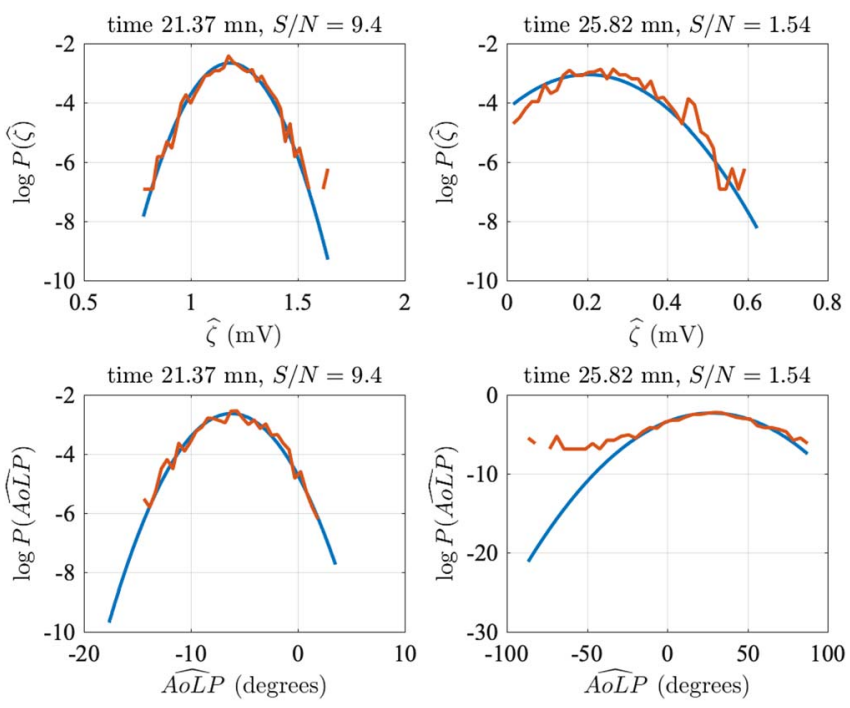

Fig. A.2. Theoretical asymptotic Gaussian probability density functions of the estimators of $\zeta$ and AoLP (blue lines, in $\log$ ) superposed to the estimated probability density functions of the estimators using the parametric bootstrap (red line, in log). The top line corresponds to $\zeta$, the bottom line to the AoLP. The left column is calculated at time $21.37 \mathrm{~min}$ for a parameter $S / N=9.4$. The right column is calculated at time $25.82 \mathrm{~min}$ for a parameter $S / N=1.54$. This clearly confirms that convergence of the estimators to a Gaussian whose theoretical parameters are calculated in the paper is relevant only for high values of $S / N$, typically greater than 4 or 5 , and that for very low $S / N$ the estimated AoLP is almost not meaningful.

parameter $\zeta$ and AoLP the theoretical asymptotic Gaussian probability density function for the estimators (blue lines) as well as the empirical histograms calculated using the boostrapped surrogates (red lines) (the log probabilities are plotted). Note that we chose for this illustration $\zeta$ instead of $\zeta_{0}$ since $\zeta$ is more difficult to estimate in terms of relative error. As discussed above, convergence is clear for a high $S / N$ for both $\zeta$ and AoLP, since the empirical fits perfectly well with the theoretically predicted (Gaussian limit). However, for a low $S / N$, we observe a clear discrepancy between the empirical and the Gaussian limit. This discrepancy is not strong for $\zeta$, but is dramatic for AoLP. Furthermore, we observe for AoLP at $S / N=1.54$ that the discrepancy is highly concentrated in the queues of the distribution, this being an explanation for the many outliers depicted around this time in the box plot of the preceding figure.

\section{Appendix B}

\section{Computation of the apparent angle of the magnetic field on the instrument}

\section{B.1 Introduction}

Computing the apparent angle of the magnetic field as seen by the polarimeter in any direction is a classical spherical geometry problem. However, it is difficult to find the development in geophysical articles or textbooks. A first development was given in the supplementary material of Lilensten et al (2016). However, this development was incomplete. Therefore, we chose to provide it here (also correcting for some typos). It is not something new, reason to write it as an appendix.

The topology of the problem is shown in Figures B.1, B.2 and B.3. In the following, we call $\Phi$ the latitude and $\Psi$ the longitude. Petit $\mathrm{Cru}$ is located in point $\mathrm{A}$, characterized by a latitude $\Phi_{A}$ and a longitude $\Psi_{A}$. The beam axis is oriented with an elevation $\varepsilon$ and an azimuth $\alpha$. The elevation is the angle with the plan which is tangent to the sphere in $A, \varepsilon=0^{\circ}$ when pointing at the horizon and $\varepsilon=90^{\circ}$ when pointing at the zenith. The azimuth is the angle with the meridian in A. It is taken equal to zero pointing North, to $90^{\circ}$ East, $180^{\circ}$ South and $270^{\circ}$ West.

Lilensten et al. (2015) have shown that the theoretical red line polarization originates from the observations at the altitude at which the observed line emission maximizes. We assume this holds also for the other lines, and we compute the projection of the magnetic field line at the altitudes $h$ listed in Table 1 . We call $H$ the point where the line of sight is at an altitude $h$, a latitude $\Phi_{H}$ and a longitude $\Psi_{H}$. If $O$ is the center of the Earth, the axis $\overline{O H}$ intersects the surface of the Earth at a point $P$ of same latitude and longitude. The vector $\overrightarrow{A H}$ carrying the line of sight has a norm $|A H|$ and is carried by the unit vector $\vec{s}$.

The frame of reference $\mathcal{R}_{O}$ at the Earth center $O$ is the usual Cartesian coordinate system, defined as follows. Axis $\vec{x}$ is in the plane of the equator with longitude $\Psi=0^{\circ}$. Axis $\vec{y}$ is in the plane of the equator with longitude $\Psi=90^{\circ}$. Axis $\vec{z}$ is vertical and points positively toward the north pole, with latitude $\Phi=90^{\circ}$.

The frame of reference $\mathcal{R}_{A}$ attached to any point $A$ on the Earth's surface is as follows. Axis $\vec{u}$ points to the zenith ( $u$ standing for up), corresponding to the altitude, positive upward. Axis $\vec{e}$ points positively toward the east in the plan tangent to the surface in $A$. Axis $\vec{n}$ points positively toward the north in the same plane.

A last set of basis vector is used as a reference frame attached to the instrument (Fig. B.2). $R_{*}=\left\{x_{*}, y_{*}, z_{*}\right\}$ where $x_{*}$ is the unit vector along the line of sight, $y_{*}$ and $z_{*}$ are in the plane of Petit Cru. When pointing north at the horizon, $y_{*}$ is pointing west and $z *$ is pointing up.

\section{B.2 First step: Finding the vector of the line of sight in $\mathcal{R}_{A}$}

Let us first compute the latitude $\Phi_{\mathrm{H}}$ and longitude $\Psi_{\mathrm{H}}$ of the observed point $H$ as seen from $A$. The radius of the Earth at point $A$ and $H$ is $R_{T}^{A}$ and $R_{T}^{H}$ respectively, and the altitude of $A$ above sea level is $a$. Finally, the distance between $A$ and $H$ is called $|A H|$ (see Fig. B.3). The generalized Pythagorus theorem gives

$$
\begin{aligned}
\left(R_{T}^{H}+h\right)^{2}= & \left(R_{T}^{A}+a\right)^{2}+|A H|^{2}-2 \times\left(R_{T}^{A}+a\right) \\
& \times|A H| \times \cos \left(\frac{\pi}{2}+\varepsilon\right) .
\end{aligned}
$$

Solving (B.1) for $|A H|$, one finds

$$
\begin{aligned}
|A H|= & -\left(R_{T}^{A}+a\right) \times \sin (\varepsilon) \\
& +\sqrt{\left(R_{T}^{A}+a\right)^{2} \times \sin (\varepsilon)^{2}+\left(R_{T}^{H}+h\right)^{2}-\left(R_{T}^{A}+a\right)^{2}} .
\end{aligned}
$$




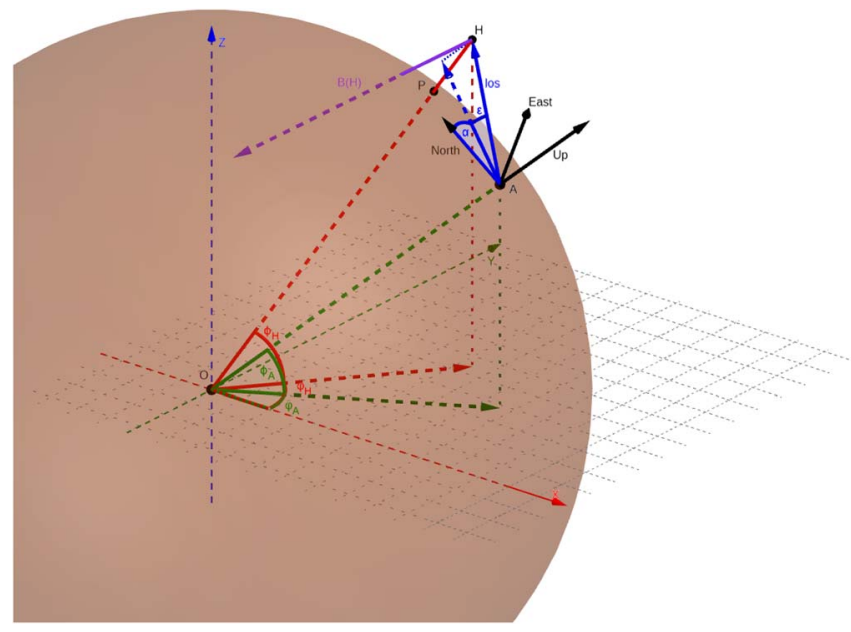

Fig. B.1. Topology of the problem. The most important points and vectors are represented. The distances are not to scale.

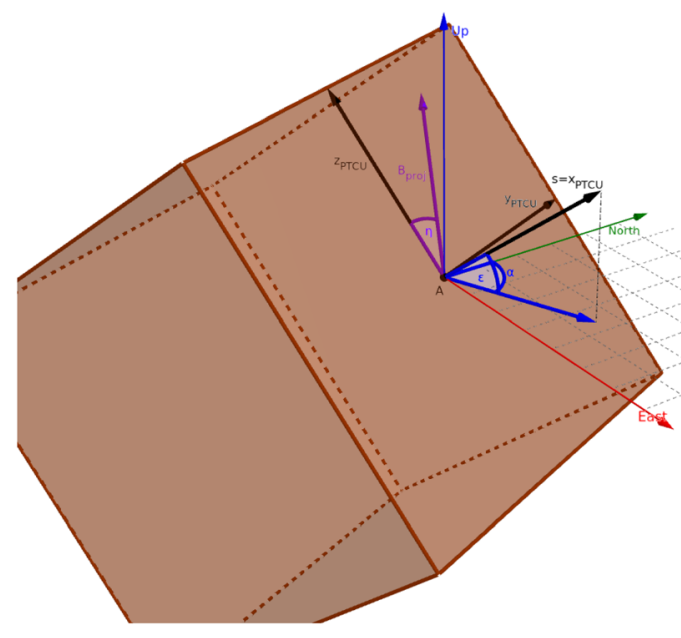

Fig. B.2. Representation of Petit $\mathrm{Cr} u$ at point $A$ with the main angles and vectors. The plane of Petit $C r u$ is represented in blue with its basis vectors $\left(R_{*}=\left\{x_{*}, y_{*}, z_{*}\right\}\right)$. $\mathcal{R}_{A}$ is composed of the three vectors $\{\mathrm{Up}$, East, North $\}$. Angles $\alpha$ and $e$ are the azimut and elevation respectively. $B_{\text {proj }}$ is the projection of the magnetic field at point $H$ on the plane of Petit Cru, and $\eta$ is the apparent angle we want to calculate.

This equation can be simplified by taking $R_{T}^{A}=R_{T}^{H}=R_{T}$ and $a=0$. Indeed, the fact that the Earth is approximated by a sphere and the variations of altitudes do not influence significantly the final result.

To find the unit vector $\vec{s}$ of the line of sight, we take the azimuth oriented positively clockwise and zero towards north. Taking the elevation into account, one gets in $\mathcal{R}_{A}$ :

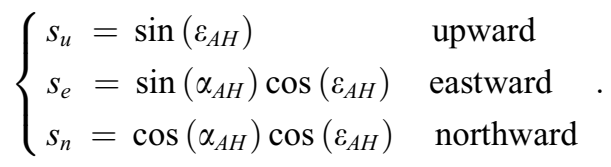

From equations (B.2) and (B.3), one deduces the vector $\overrightarrow{A H}$ :

$$
\overrightarrow{A H}=|A H| \vec{s} .
$$

\section{B.3 Second step: Finding $\boldsymbol{H}$ coordinates}

The geometry of the problem is displayed in Figure B.1. Petit Cru may be positioned anywhere on Earth. The first step is to express every vector in the same reference frame. Let us first express $\overrightarrow{s_{A}}$, the unit vector of our line of sight in $\mathcal{R}_{O}$. The transformation matrix to pass from the reference frame $\mathcal{R}_{A}$ at point $A$ to $\mathcal{R}_{O}$ at point $O$ is:

$$
R_{A \rightarrow O}=\left(\begin{array}{ccc}
\cos \left(\Phi_{A}\right) \cos \left(\psi_{A}\right) & -\sin \left(\psi_{A}\right) & -\sin \left(\Phi_{A}\right) \cos \left(\psi_{A}\right) \\
\cos \left(\Phi_{A}\right) \sin \left(\psi_{A}\right) & \cos \left(\psi_{A}\right) & -\sin \left(\Phi_{A}\right) \sin \left(\psi_{A}\right) \\
\sin \left(\Phi_{A}\right) & 0 & \cos \left(\Phi_{A}\right)
\end{array}\right)
$$

Its inverse $R_{O \rightarrow A}$, to pass from $\mathcal{R}_{O}$ to $\mathcal{R}_{A}$ is the transposed matrix of $R_{A \rightarrow O}$. Of course, this transformation works for every points on the globe and is very practical to express all vectors in a unique reference frame. For example, $\overrightarrow{s_{A}}$ can be expressed as follows in $\mathcal{R}_{0}$ :

$$
\overrightarrow{s_{O}}=R_{A \rightarrow O} \overrightarrow{s_{A}} .
$$

Another important step is to express the coordinates of a point $A$ on the surface of the Earth, with latitude $\Phi_{A}$ and longitude $\Psi_{A}$ in $\mathcal{R}_{O}$. This is the same as expressing the vector $\overrightarrow{O A}$ in $\mathcal{R}_{O}$ as follows:

$$
\overrightarrow{O A}=R_{T}^{A}\left(\begin{array}{c}
\cos \left(\Phi_{A}\right) \cos \left(\Psi_{A}\right) \\
\cos \left(\Phi_{A}\right) \sin \left(\Psi_{A}\right) \\
\sin \left(\Phi_{A}\right)
\end{array}\right)
$$

Using the relation $\overrightarrow{O H}=\overrightarrow{O A}+|A H| \vec{s}$, we can develop on the three axis $\{x, y, z\}$ of $\mathcal{R}_{O}$ using equations (B.6) and (B.7):

$$
\overrightarrow{O H}=R_{T}^{A}\left(\begin{array}{c}
\cos \left(\Phi_{A}\right) \cos \left(\Psi_{A}\right) \\
\cos \left(\Phi_{A}\right) \sin \left(\Psi_{A}\right) \\
\sin \left(\Phi_{A}\right)
\end{array}\right)+|A H| \overrightarrow{s_{O}} .
$$

From the vector $\overrightarrow{O H}$ as expressed using equation (B.8), we can easily deduce the latitude $\Phi_{H}$ and the longitude $\Psi_{H}$ of the point $H$, passing from the Cartesian coordinates to the spherical coordinates:

$$
\left\{\begin{array}{l}
\Phi_{H}=\arctan \left(\frac{O H_{z}}{\sqrt{O H_{x}^{2}+O H_{y}^{2}}}\right) \\
\Psi_{H}=\arctan \left(\frac{O H_{y}}{O H_{x}}\right)
\end{array} .\right.
$$

\section{B.4 Apparent angle of observation}

The next question to solve is that of the apparent angle of the magnetic field in $H$ (latitude $=\Phi_{H}$, longitude $=\Psi_{H}$, heigh $=h$ ) projected on Petit Cru. The configuration is shown 


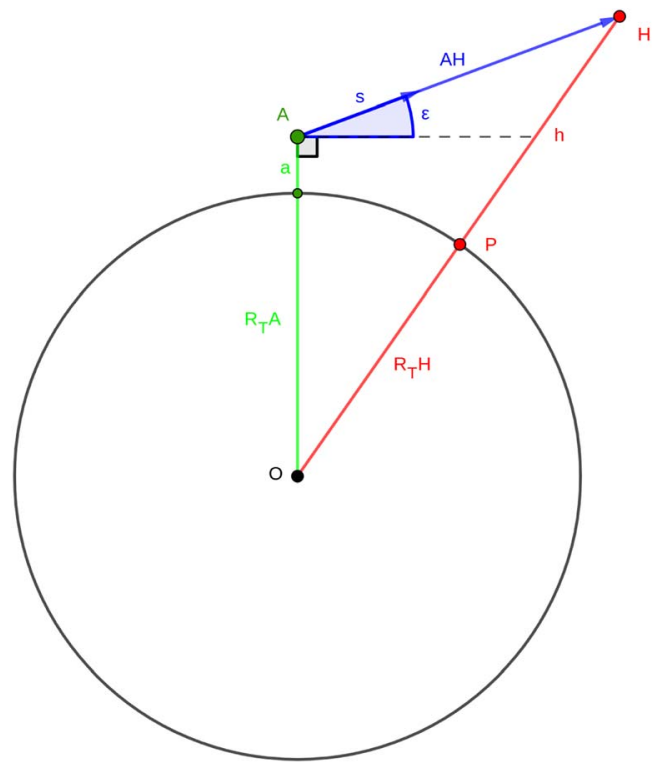

Fig. B.3. Representation of an observation to find the distance $A H$.

in Figure B.1. We tested both the CHAOS-6 (Finlay et al., 2016) and IGRF (Thébault et al., 2015) geomagnetic geomagnetic field model to get the magnetic field $B_{\mathrm{H}}$ at the observed point $H$. Both give very close results, with differences too small to be visible in our work. This is why we only show results using CHAOS-6 in this paper. We calculate from CHAOS-6 the three coordinates of the magnetic field $\left\{B_{u}, B_{e}, B_{n}\right\}$ in the frame of $H$, characterized by three axis $\left\{u_{H}, e_{H}, n_{H}\right\}$. It is necessary to rotate these values in the reference frame of $A$, characterized by $\left\{u_{A}, e_{A}, n_{A}\right\}$. The first rotation expresses the magnetic field $B_{H}$ in $\mathcal{R}_{O}$ using equation (B.5):

$$
\overrightarrow{B_{O}}=R_{H \rightarrow O} \overrightarrow{B_{H}} \text {. }
$$

The second rotation brings the field in $\mathcal{R}_{A}$ :

$$
\begin{aligned}
\overrightarrow{B_{A}} & =R_{O \rightarrow A} \overrightarrow{B_{O}} \\
& =R_{O \rightarrow A} R_{H \rightarrow O} \overrightarrow{B_{H}} .
\end{aligned}
$$

Computing the magnetic angle seen by Petit $\mathrm{Cr} u$ is performed through a last set of rotations that brings the magnetic field $\overrightarrow{B_{A}}$ in the frame of reference of the polarimeter $R_{*}=$ $\left\{x_{*}, y_{*}, z_{*}\right\}$. The rotation matrix is:

$$
R_{* \rightarrow A}=\left(\begin{array}{ccc}
\sin (\varepsilon) & 0 & \cos (\varepsilon) \\
\cos (\varepsilon) \sin (\alpha) & -\cos (\alpha) & -\sin (\varepsilon) \sin (\alpha) \\
\cos (\varepsilon) \cos (\alpha) & \sin (\alpha) & -\sin (\varepsilon) \cos (\alpha)
\end{array}\right)
$$

Again, its inverse matrix to pass from the reference frame of $A$ to the reference frame of Petit $C r u$ is its transposed matrix $R_{A \rightarrow *}$. We can now write the vector of the magnetic field $\overrightarrow{B_{*}}$ as seen by the polarimeter:

$$
\begin{aligned}
\overrightarrow{B_{*}} & =R_{A \rightarrow *} \overrightarrow{B_{A}} \\
& =R_{A \rightarrow *} R_{O \rightarrow A} R_{H \rightarrow O} \overrightarrow{B_{H}} .
\end{aligned}
$$

Then the apparent angle of the magnetic field $\eta$ is simply defined by:

$$
\tan (\eta)=\frac{B_{*, y}}{B_{*, z}}
$$

\section{Appendix C}

\section{Computation of the apparent angle of Rayleigh scattered light from a localized source}

We use the same mathematical tools and notation for this calculation than for the computation of the apparent angle of the magnetic field on the instrument in Appendix B.

The instrument is placed at point $A$, looking in a given direction with a unit vector $\vec{S}$. The point source is placed at point $S$, with the associated unit vector $\overrightarrow{A S}$. Two reference frames are used. First $\mathcal{R}_{A}$ attached to point $A$ on the Earth's surface is defined by three unit vectors $(\vec{u}, \vec{e}, \vec{n})$ as follows:

- Axis $\vec{u}$ points to the zenith ( $u$ standing for up). It corresponds to the altitude axis, positive upward.

- Axis $\vec{e}$ points positively towards East in the plane tangent to the Earth's surface in $A$.

- Finally, axis $\vec{n}$ points positively towards North in the same horizontal plane.

The second reference frame $\mathcal{R}_{*}$, attached to the instrument, is defined by three unit vectors $\left(\vec{x}_{*}, \vec{y}_{*}, \vec{z}_{*}\right)$, where $\vec{x}_{*}$ is along the line of sight, while $\vec{y}_{*}$ and $\vec{z}_{*}$ are perpendicular to it (in the plane of the Petit Cru polarimeter, see Fig. B.2). When pointing North at the horizon, $\vec{y}_{*}$ is pointing west and $\vec{z}_{*}$ is pointing up.

We characterize the line of sight, of unit vector $\vec{s}$ in $\mathcal{R}_{A}$, from the azimuth $\alpha_{s}$ and elevation $\varepsilon_{s}$ of the instrument:

$$
\begin{aligned}
s_{u} & =\sin \left(\varepsilon_{s}\right) \quad \text { upward, } \\
s_{e} & =\sin \left(\alpha_{s}\right) \cos \left(\varepsilon_{s}\right) \quad \text { eastward, } \\
s_{n} & =\cos \left(\alpha_{s}\right) \cos \left(\varepsilon_{s}\right) \text { northward. }
\end{aligned}
$$

We also characterize the vector $\overrightarrow{A S}$ in $\mathcal{R}_{A}$ from the corresponding azimuth $\alpha_{\text {src }}$ and elevation $\varepsilon_{\text {src }}$ of the apparent direction of the source from $A$ :

$$
\begin{array}{ll}
A S_{u}=\sin \left(\varepsilon_{A S}\right) & \text { upward, } \\
A S_{e}=\sin \left(\alpha_{A S}\right) \cos \left(\varepsilon_{A S}\right) & \text { eastward, } \\
A S_{n}=\cos \left(\alpha_{A S}\right) \cos \left(\varepsilon_{A S}\right) & \text { northward. }
\end{array}
$$

From Rayleigh theory (e.g. Bohren and Huffman, 2008), the angle of polarization of the scattered light is perpendicular to the scattering plane $P$ formed by the incoming light beam and the scattered light beam (see Fig. C.1). This plane is sufficient to define the angle of polarization of the scattered light. By construction, points $A$ (the instrument) and $S$ (the source) and the line of sight are all part of plane $P$. This plane contains any line joining the source and the line of sight, such that the point of 


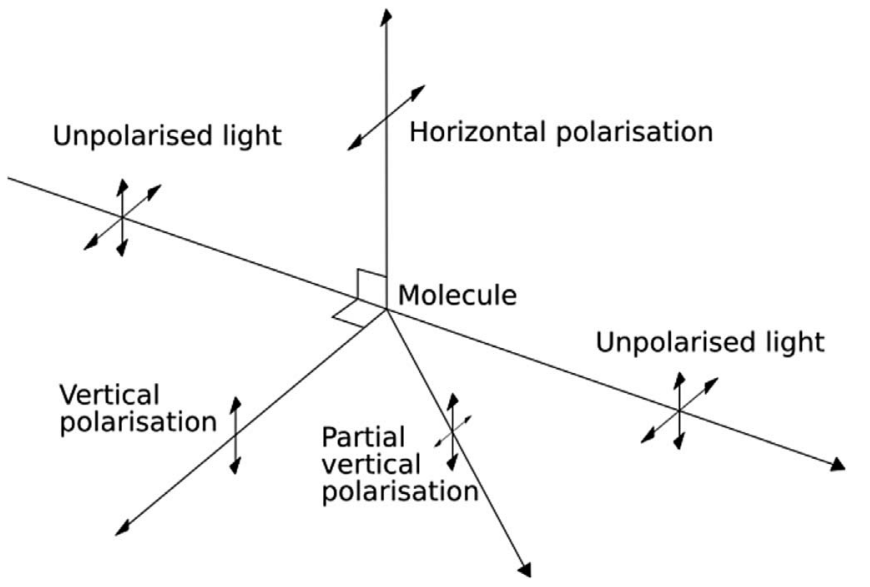

Fig. C.1. Light polarisation by Rayleigh scattering. Light scattered at an angle in the horizontal (vertical) plane will be polarised vertically (horizontally). Note that the DoLP of scattered light is maximal for a $90^{\circ}$ scattering angle, and minimal for a $0^{\circ}$ scattering angle. However, we do not need to know the DoLP for the present purpose.

scattering along the line of sight does not matter for the computation of the polarization angle. Furthermore, the line joining $A$ and $S$ is also contained in this plane, so that we are also insensitive (for the present purpose) to the distance from the source to the instrument.

$\vec{S}$ and $\overrightarrow{A S}$ are both in the plane $P$. We can use their vector product to get the direction $\vec{p}$ of the polarized light in $\mathcal{R}_{A}$ :

$$
\vec{p}=\vec{s} \wedge \overrightarrow{A H}=\left(\begin{array}{c}
s_{e} A S_{n}-s_{n} A S_{e} \\
s_{n} A S_{u}-s_{u} A S_{n} \\
s_{u} A S_{e}-s_{e} A S_{u}
\end{array}\right)
$$

We can pass $\vec{p}$ in the reference frame of the instrument $\mathcal{R}_{*}$ by using the rotation matrix

$$
R_{A \rightarrow *}=\left(\begin{array}{ccc}
\sin (\varepsilon) & \cos (\varepsilon) \sin (\alpha) & \cos (\varepsilon) \cos (\alpha) \\
0 & -\cos (\alpha) & \sin (\alpha) \\
\cos (\varepsilon) & -\sin (\varepsilon) \sin (\alpha) & -\sin (\varepsilon) \cos (\alpha)
\end{array}\right)
$$

The angle of polarization of the scattered light from point source $S$ is then $\gamma$, simply defined as

$$
\tan (\gamma)=\frac{p_{*, y}}{p_{*, z}} .
$$

Cite this article as: Bosse L, Lilensten J, Gillet N, Rochat S, Delboulbé A, et al. 2020. On the nightglow polarisation for space weather exploration. J. Space Weather Space Clim. 10, 35. 